\title{
Biologically Plant-Based Pigments in Sustainable Innovations for Functional Textiles - The Role of Bioactive Plant Phytochemicals
}

\author{
Alka Madhukar Thakker* and Danmei Sun \\ School of Textiles and Design, Heriot-Watt University, UK
}

*Corresponding author: Alka Madhukar Thakker, School of Textiles and Design, Heriot-Watt University, TD1 3HF, UK. Email: amt5@hw.ac.uk

Received Date: March 29, 2021

Published Date: June 01, 2021

\begin{abstract}
The fossil-based emissions and effluents from the textile wet processing units have acute implications on the environment and human wellbeing. Therefore, a paradigm shift towards sustainable alternative materials and technology has become imperative. Thus, the overview herein emphasizes the sustainable chemistry that ubiquitously exists to propel ecological textile processing with herbal biomaterials. The review paper succinctly accentuates the efficient qualities of bioactive plant pigments for functional textiles. The paper also outlines the sustainable method of processing with plant phytochemicals. The eco-friendly herbal materials as an excellent example of Biomimicry is presented. Likewise, the multi-disciplinary research approach is discussed herein to refute global dilemmas and impel holistic ecological outcomes. The circular materials for the circular economy are detailed.
\end{abstract}

Keywords: Sustainable chemistry; Bioactive pigments; Herbal colors; Functional fabrics; Sustainable textiles

\section{Introduction}

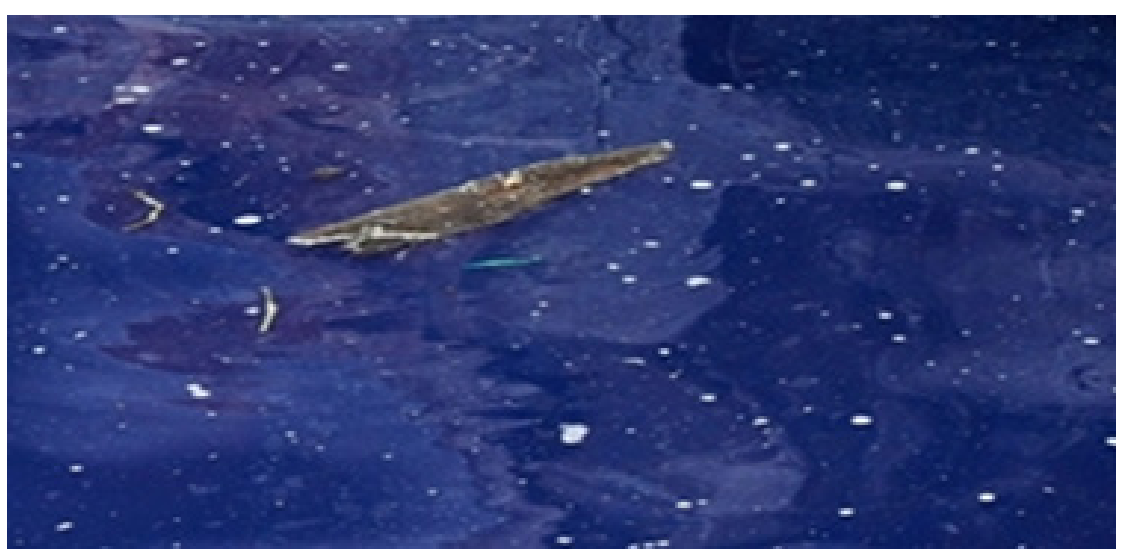

Figure 1: The dilemma of Citarum river, Indonesia, colored blue due to toxic synthetic dye effluents discharged from the surrounding textile wet processing units [4]. 
Greenpeace international initiated Detox My Fashion campaign in 2011 to ensure that the clothing was free from hazardous chemicals from make to finish [1]. For example, their research findings on the Citarum river contamination refer to Figure 1. concluded it to be loaded with chromium and copper-containing dyes of $0,005 \mathrm{mg} / \mathrm{l}$ and heavy metals from textile mills resulting in low levels of dissolved oxygen (DO) in the water of $2.45 \mathrm{mg} / \mathrm{l}$. The minimum required DO is $4 \mathrm{mg} / \mathrm{l}$ to sustain aquatic life. It took a heavy toll on aquatic life with $60 \%$ of fish dead also affecting surrounding flora and fauna [2]. They further launched Zero Discharge of Hazardous Chemicals (ZDHC) wherein they predominantly accentuate implementing eco-smart process and safe Chemicals in textile processing units. ZDHC formulated a Manufacturing Restricted Substances List, or M-RSL, which appeal to mandatorily eliminate 11 priority hazardous chemical groups refer to https://mrsl.roadmaptozero.com/MRSL2_0. It prohibits the use of heavy metals namely chromium, copper etcetera also alkylphenol ethoxylates (APEs), per- and polyfluorinated chemicals
(PFCs), and phthalates. The list of restricted carcinogenic dyes namely azo, disperse etcetera, and anti-microbials namely triclosan etcetera are detailed in the ZDHC M-RSL [3]. This impels the research and development of alternative biodegradable and renewable sources of natural materials for the application of textile substrates for coloration and functionality namely anti-microbial, analgesic, antioxidant etcetera, hence the instigated review paper (Figure 1).

Several authors have taken a unique approach towards sustainability in textiles however the overview herein focuses on plant-based renewable and biodegradable points of sources for sustainability in textiles.

\section{Ecological Processing of Plant Phytochemical's}

For green consequences, it is crucial to adopt processing tools and techniques that would be low on water and energy demand. The current sustainability challenges faced by the textile industry are summarized in Table 1.

Table 1: Sustainability challenges confronted by the textile industry [5].

\begin{tabular}{|c|c|}
\hline Stages & Best practices to implement \\
\hline Fiber/fabric processing & Minimum steps, reuse, recycle, reduce consumption of resources. \\
\hline Scouring & \multirow{3}{*}{ Utilize Plant-based materials, combine the steps, and lower the effluent pollution index } \\
\hline Desizing & \\
\hline Bleaching & \\
\hline Dyeing & $\begin{array}{l}\text { Plant-based natural dyes and dye assistants, simplify synthetic dyeing with the right } \mathrm{pH} \text {, auxiliary, enzymes and } \\
\text { reduce the effluent quantity and toxicity. }\end{array}$ \\
\hline Printing & Formaldehyde-free, urea free, non-volatile water-based inks, thickeners, and solvents. \\
\hline Pre- and post-treatment & Non-metallic, non-ionic, biodegradable. \\
\hline $\begin{array}{l}\text { Surface modification's and } \\
\text { treatments }\end{array}$ & Mechanical, nontoxic, simple. Techniques that save energy and water usage. \\
\hline
\end{tabular}

The overview particularly centers on renewable plant-based natural materials therefore elaborates on the sustainable procedure for suitable application of herbs on textile substrates. There is a growing body of literature that recognizes the importance of sustainability in textile wet processing units [5].

Under the sustainability agenda, previous studies make a noteworthy revelation, the natural plant phytochemicals responsible for functionality and coloration on a textile substrate are preserved at low infusion temperatures, low dilution, and in plain water as a solvent [5-8]. Therefore, greater functional benefits and original color are achieved with minimal heat concoctions of herbal leaves, stems, barks, buds, and flowers. At the same time being low on energy, water, and synthetic solvent demands would impel sustainable results.

The naturally occurring plant phytochemicals namely polyphenolic compounds for example quercetin, kaempferol, catechins, and anthocyanins others contained in cloves, green tea, mango turmeric, kattha others responsible for anti-oxidant, anti-microbial, anti-inflammatory functionality are very sensitive to heat treatment and are expended on boiling. The temperature of the water of 30 to $60{ }^{\circ} \mathrm{C}$ is most advantageous for maintaining original color stability and functional phytochemical contents [6-8]. Subsequently, low temperature and overnight extraction or dyeing were concluded to be conducive for the processing of natural fiber/ fabrics with natural herbs.

In the same vein, reheating, and prolonged heating at a temperature beyond $60^{\circ} \mathrm{C}$ perishes the phytochemicals for example $62 \%$ of the phenolic acids would get destroyed in 2 minutes of 100 ${ }^{\circ} \mathrm{C}$ heat aka boiling temperature [9]. The process is simple and clean involving no emissions and effluents. Hence, the ecological devised process which concurrently adheres to Sustainable developmental goals (SDGs) [10]. The method developed propels optimal functionality and original color stability. For example, Figures 2,3, and 4 elaborates on the surplus raw materials, ecological processing implemented in research experiments, and likewise the obtained sustainable results depicted in images [8]. 


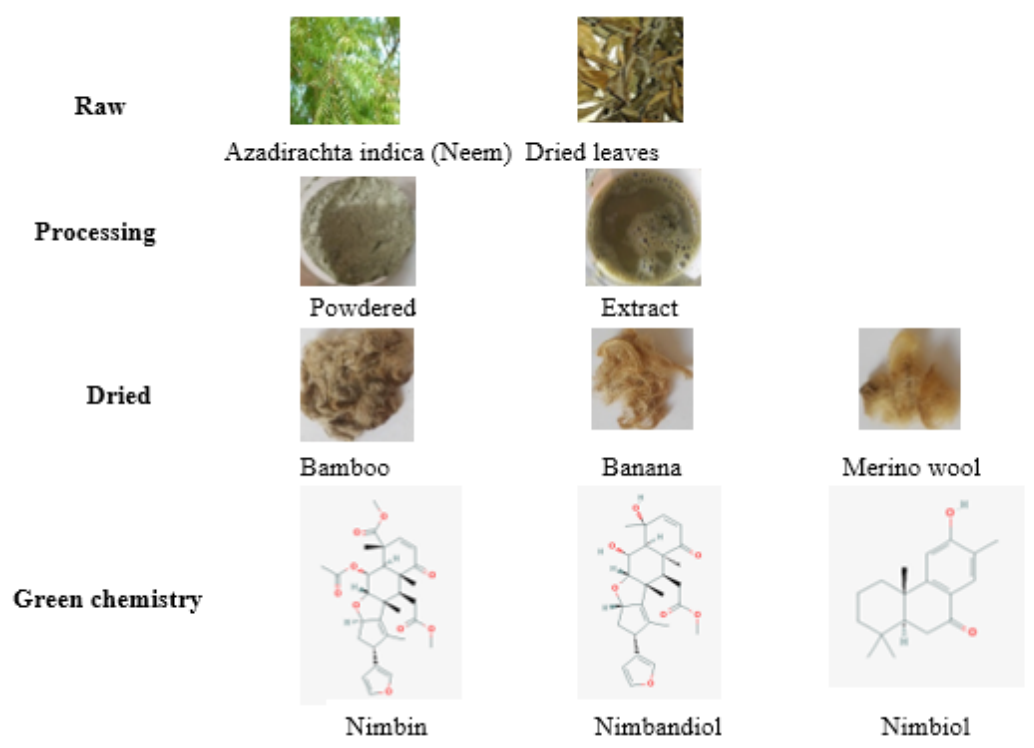

Figure 2: Sustainable processing of bamboo, banana, and merino wool with Azadirachta indica (Neem) [8].

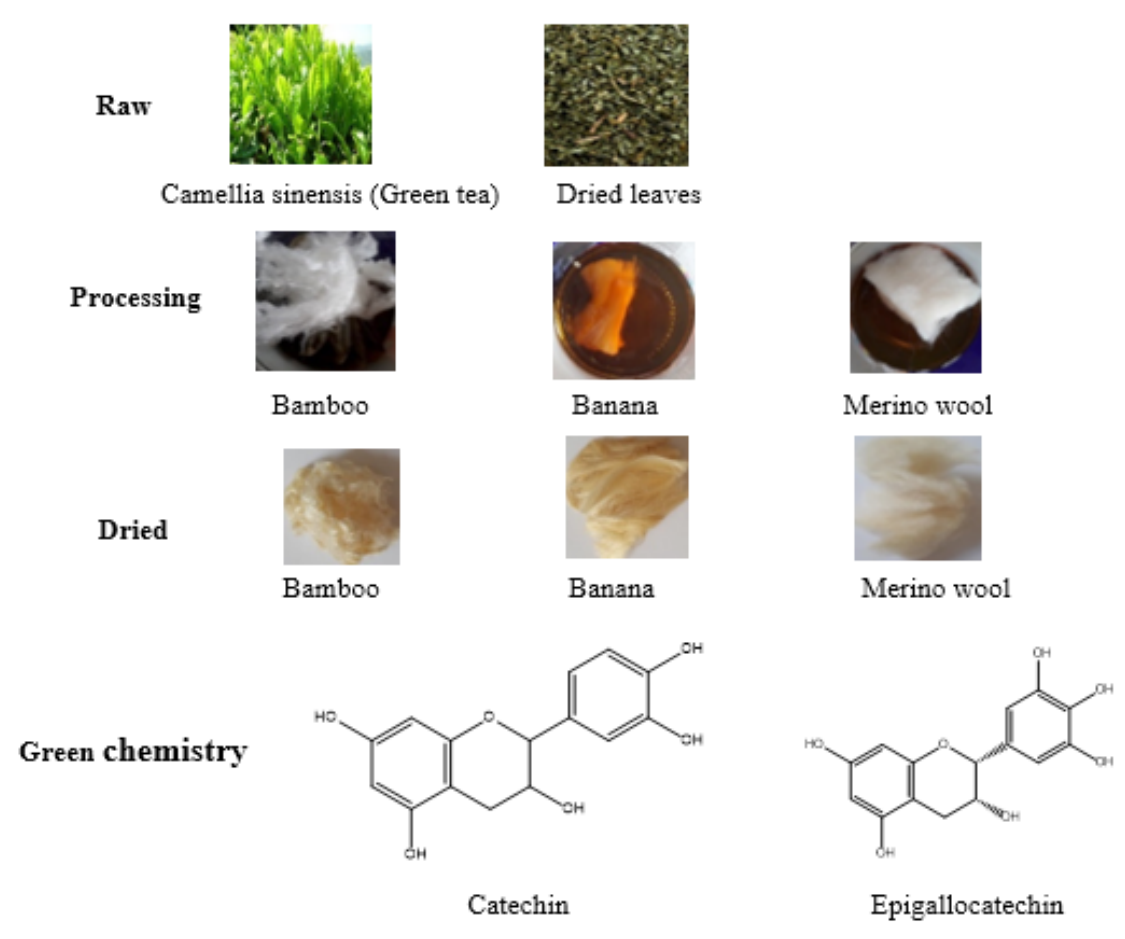

Figure 3: Sustainable processing of bamboo, banana, and merino wool with Camellia sinensis (Green tea) [8]. 


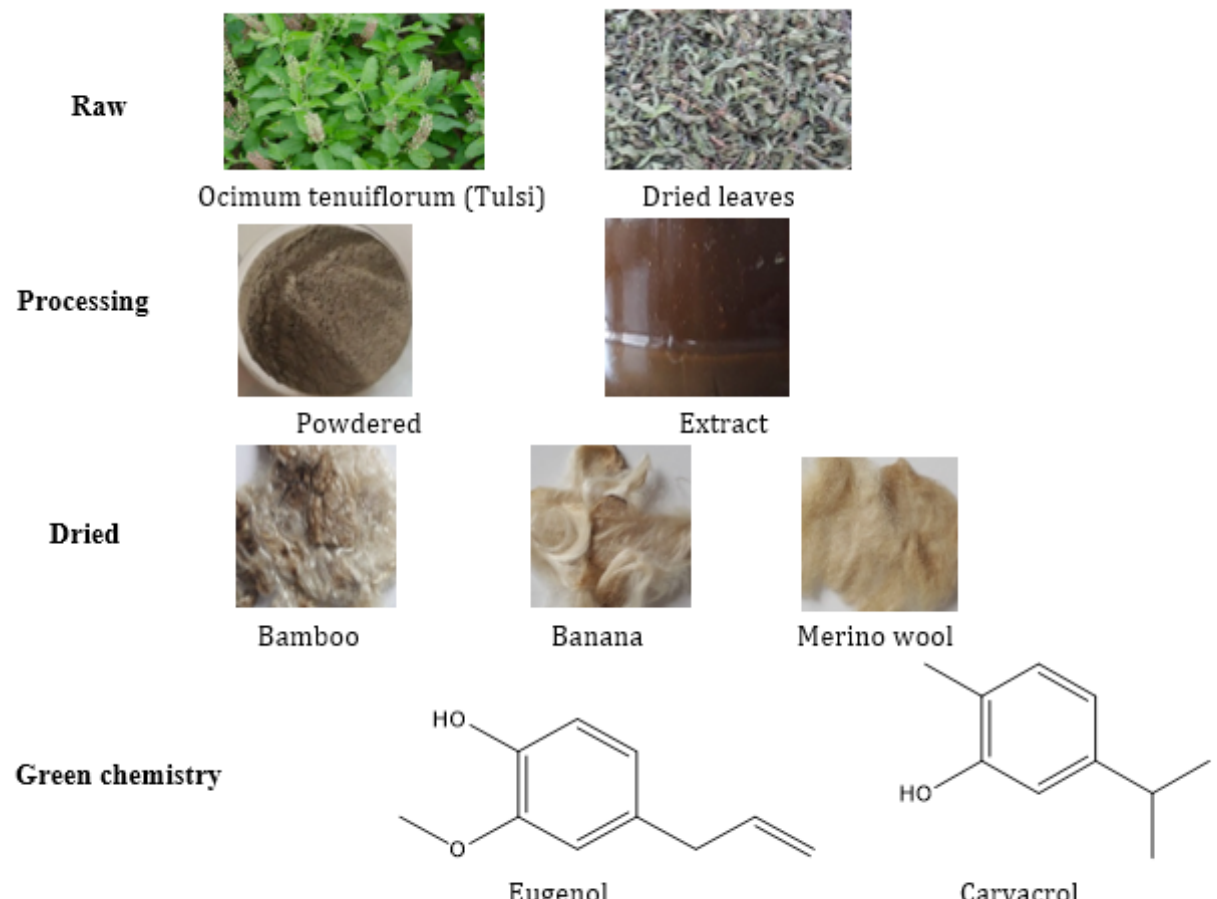

Figure 4: Sustainable processing of bamboo, banana, and merino wool with Ocimum tenuiflorum (Tulsi) [8] Functionality: Anti-inflammation, anti-microbial, anti-oxidant, kind to skin and environment.

The green chemistry inherently manifested by herbal materials, the functionality of herbs, and color obtained on natural textile fibers namely bamboo fibers, banana fibers, and merino wool fibers are shown in Figures 2,3, and 4. It is remarkable to note that Chemical Structures of Bioactive Plant Phytochemicals sustainable natural plant fibers namely bamboo fibers, banana fibers, and merino wool fibers as cited above have inherently high compatibility with plant pigments and therefore enhanced functionality.

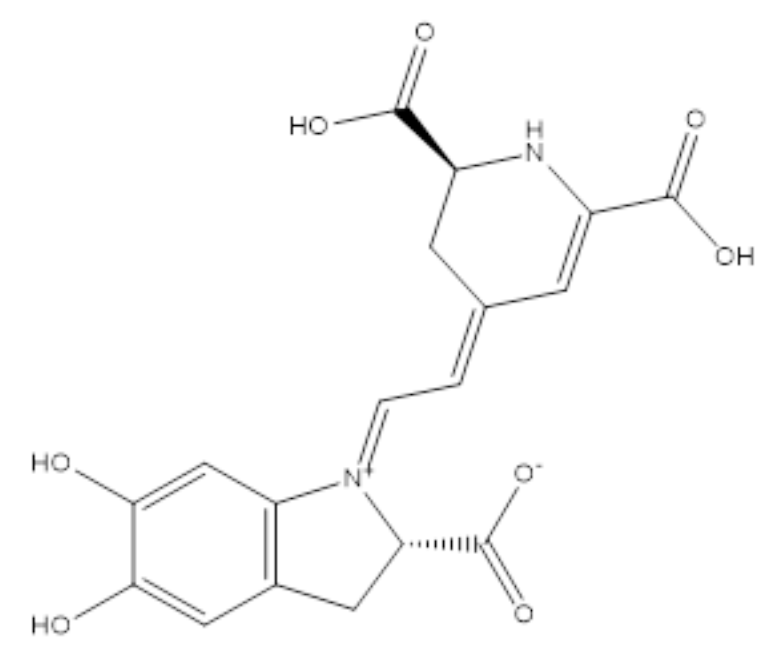

Figure 5: Betanidin [11].

The green chemistry is highlighted herein for ecological benefits all the more the scientific research investigations accomplished on natural colors from plants indicate no possible health treat or toxicity to humans and animals [11]. The chemical modification of synthetic chemistry to ecological green chemistry is mandatory for the paradigm shift towards sustainability in the textile industry. 
The classification of chemical structures of plant phytochemicals is presented in Figures 5 to 84 [12-15]. The most relevant to the textile substrate application for coloration and functionality is specified here.

Anthraquinones, a yellow crystalline ketone with chemical formula C14H8O2 often derived from anthracene.

Anthracene is a crystalline tricyclic aromatic hydrocarbon with the chemical formula C14H10. For example, Indian mulberry consists of two anthraquinones Soranjidiol and Morindone $[13,14]$, refer to Figure 12 to 36.

Betalains, a nitrogen-containing anthocyanin constituting the chief coloring matter of garden beets having a chemical formula, $\left(\mathrm{CH}_{3}\right)_{3} \mathrm{~N}^{+}-\mathrm{CH}_{2} \mathrm{CO}_{2}^{-}$[13-14], refer to Figure 5 and Figure 37,38, and 39.

Indoles, a heteroaromatic compound with fused benzene and pyrrole rings; chemical formula: $\mathrm{C}_{8} \mathrm{H}_{7} \mathrm{~N}$. it is a crystalline alkaloid compound that is a decomposition product of proteins containing tryptophan [13-14]. For example, Indigo containing Indigotin [12], refer to Figure 40.

Quinone methides, a quinone derivative with a methylene group in place of one of the oxygen atoms. For example, Red sandalwood containing santalins A and B [13-14], refer Figures 41 to 44 .

Pyrones, either of two isomeric carbonyl compounds $\mathrm{C} 5 \mathrm{H} 4 \mathrm{O} 2$ derived from pyran. The pyrones are keto derivatives of the pyrans; the commoner isomer, " $\gamma$-pyrone", is a colorless, basic, crystalline solid, $\mathrm{CH}=\mathrm{CHOCH}=\mathrm{CHCO}$ [13-14]. For example, mangostin contained in pitch apple [12], refer to Figure 45 to 48.

Benzophenones, a colorless crystalline ketone C13H100 [13], for example, maclurin contained in jackfruit [12], refer to Figures 49 and 50.

Alkaloids are any of numerous usually colorless, complex, and bitter organic bases (such as morphine or caffeine) containing nitrogen and usually oxygen that occur especially in seed plants and are typically physiologically active $[13,14]$. For example, Cryptolepine contained in gangamau [12], refer to Figure 6 and Figure 51 to 58.<smiles>CN1c2ccccc2C=C2Nc3ccccc3C21</smiles>

Figure 6: Cryptolepine [11].

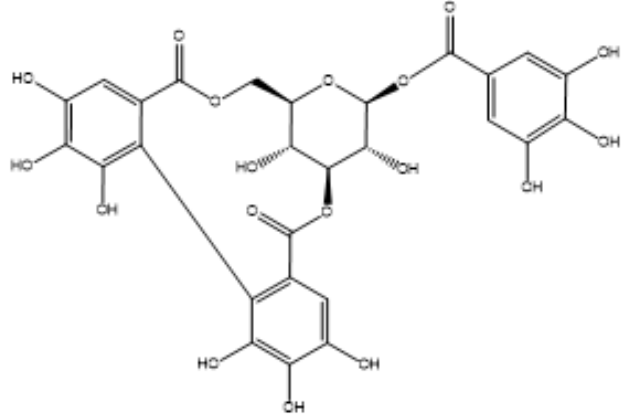

Figure 7: Corilagin [11].

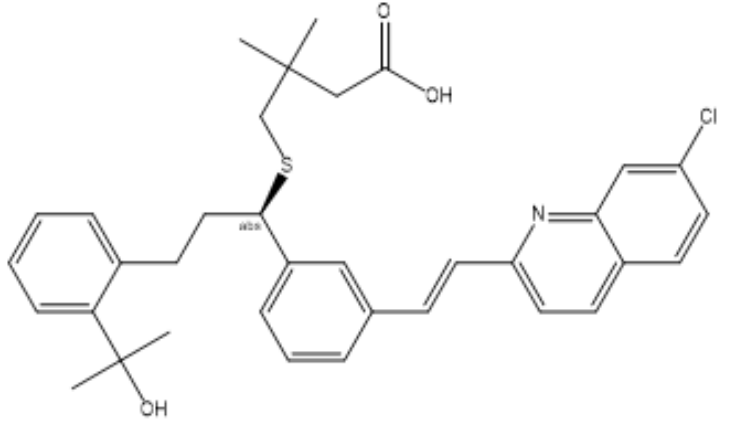

Figure 8: Chebulagic acid [11].
Tannins are a yellowish or brownish bitter-tasting organic substance present in some galls, barks, and other plant tissues, consisting of derivatives of gallic acid. Tannin is any of the various soluble astringent complex phenolic substances of plant origin used especially in tanning leather and dyeing textiles, manufacturing ink, clarifying wine and beer, and in medicine [13,14]. For example, a bedda nut tree contains corilagin, chebulinic acid, chebulic, and chebulagic acid [12], refer to Figures 7 and 8, also refer to Figures 59 to 84

Catechin is a crystalline flavonoid compound that is the major constituent of catechu. It is a phenol with chemical formula, $\mathrm{C}_{15} \mathrm{H}_{14} \mathrm{O}_{6}$ [13]. It includes epicatechin, gallocatechin, and epigallocatechin. For example, cutch tree, gambier bush, dye yam, and betel palm containing tannins catechins [12], refer to Figures 9,10 and 11. 
<smiles>Oc1cc(O)c2c(c1)OC(c1ccc(O)c(O)c1)C(O)C2</smiles>

Figure 9: Catechin [11].<smiles>Oc1cc(O)c2c(c1)O[C@H](c1cc(O)c(O)c(O)c1)[C@H](O)C2</smiles>

Figure 10: Gallocatechin [11].<smiles>Oc1cc(O)c2c(c1)O[C@H](c1cc(O)c(O)c(O)c1)[C@H](O)C2</smiles>

Figure 11: Epigallocatechin [11].

Thus, massive literature is available on the characterization of plant phytochemicals as tabulated above. The chemical structures of each are detailed as follows (Table 2) (Figures 12-84).

Table 2: Characterization of biological pigments from plants $[12,16,17]$.

\begin{tabular}{|c|c|c|c|c|}
\hline $\begin{array}{c}\text { Anthraquinones } \\
\text { In Polygonaceae } \\
\text { And Rhamnaceae } \\
\text { Figure } 12\end{array}$ & Madders, Stiff Marsh Bedstraws & Roots & Red & $\begin{array}{l}\text { Anti-Microbial, Antioxidant, Anti-Inflam- } \\
\text { matory }\end{array}$ \\
\hline $\begin{array}{c}\text { Pseudopurpurin } \\
\text { Figure } 13\end{array}$ & Dyer's Madder - Rubia Tinctorum & Roots & Purple Red & Anti-Microbial, Antioxidant, Anti-Inflammatory \\
\hline $\begin{array}{l}\text { Chrysophanol } \\
\text { Figure } 14\end{array}$ & $\begin{array}{l}\text { Rhubarbs, Docks, Garden Sorrels, Yellow Dock, And } \\
\text { Rumex Spp. - R. Alpinus, R. Patientia, R. Crispus }\end{array}$ & $\begin{array}{l}\text { Rhizomes } \\
\text { and Roots }\end{array}$ & Red & Anti-Microbial, Antioxidant, Anti-Inflammatory \\
\hline $\begin{array}{l}\text { Aloe-Emodin } \\
\text { Figure } 15\end{array}$ & $\begin{array}{l}\text { Aloes, Rhubarbs - Rheum Spp. - Himalayan Rhu- } \\
\text { barbs, Buckthorns - Rumex Spp. }\end{array}$ & $\begin{array}{l}\text { Leaves, } \\
\text { Rhizomes, } \\
\text { Bark }\end{array}$ & Red & Anti-Microbial, Antioxidant, Anti-Inflammatory \\
\hline $\begin{array}{c}\text { Rhein } \\
\text { Figure } 16\end{array}$ & $\begin{array}{l}\text { Morinda Angustifolia, Rhubarbs - Rheum undula- } \\
\text { tum, Rheum palmatum, Cassia reticulata }\end{array}$ & Stems & Red & Anti-Microbial, Antioxidant, Anti-Inflammatory \\
\hline $\begin{array}{c}\text { Rheidins A, B, C } \\
\text { Figure 17, } 18 \text { \& } 19\end{array}$ & $\begin{array}{l}\text { Rhubarbs - Rheum Spp., Alder Buckthorns - Fran- } \\
\text { gula alnus }\end{array}$ & $\begin{array}{l}\text { Rhizomes, } \\
\text { Bark }\end{array}$ & Red & Anti-Microbial, Antioxidant, Anti-Inflammatory \\
\hline $\begin{array}{l}\text { Emodin } \\
\text { Figure } 20\end{array}$ & $\begin{array}{c}\text { Rhubarbs, Docks, Evergreen Buckthorns, Pitti, } \\
\text { Fabaceae (Cassia Spp.) }\end{array}$ & $\begin{array}{l}\text { Roots, } \\
\text { Barks }\end{array}$ & Red & Anti-Microbial, Antioxidant, Anti-Inflammatory \\
\hline $\begin{array}{l}\text { Physcion } \\
\text { Figure } 21\end{array}$ & $\begin{array}{c}\text { Rhubarbs, Docks, Pitti - Ventilago Madraspatana, } \\
\text { Rubia cordifolia, Buckthorns }\end{array}$ & $\begin{array}{c}\text { Rhizomes, } \\
\text { Roots, } \\
\text { Stems- } \\
\text { Bark }\end{array}$ & Red & Anti-Microbial, Antioxidant, Anti-Inflammatory \\
\hline $\begin{array}{l}\text { Alaternin } \\
\text { Figure } 22 \\
\end{array}$ & $\begin{array}{c}\text { Rhamnus alaternus L. } \\
\text { R. Cathartica }\end{array}$ & Bark & Red & Anti-Microbial, Antioxidant, Anti-Inflammatory \\
\hline $\begin{array}{l}\text { Islandicin } \\
\text { Figure } 23\end{array}$ & Pitti, V. Calyculata, Kniphofia Spp. & Root Bark & Red & Anti-Microbial, Antioxidant, Anti-Inflammatory \\
\hline
\end{tabular}




\begin{tabular}{|c|c|c|c|c|}
\hline $\begin{array}{l}\text { Xanthorin } \\
\text { Figure } 24\end{array}$ & $\begin{array}{l}\text { Ventilago madraspatana - Pitti, V. Calyculata, Berch- } \\
\text { emia floribunda }\end{array}$ & Root Bark & Red & Anti-Microbial, Antioxidant, Anti-Inflammatory \\
\hline $\begin{array}{l}\text { Ventinones A and B } \\
\text { Figure } 25\end{array}$ & Pitti & Root Bark & Red & Anti-Microbial, Antioxidant, Anti-Inflammatory \\
\hline Calyculatone & V. Calyculata & Root Bark & Red & Anti-Microbial, Antioxidant, Anti-Inflammatory \\
\hline $\begin{array}{l}\text {-Anthraquinones In } \\
\text { Rubiaceae }\end{array}$ & $\begin{array}{l}\text { Rubia tinctorum, Rubia peregrina, Morinda citrifo- } \\
\text { lia, Morinda umellata }\end{array}$ & Roots & Red & Anti-Microbial, Antioxidant, Anti-Inflammatory \\
\hline $\begin{array}{l}\text { Alizarin } \\
\text { Figure } 26\end{array}$ & $\begin{array}{l}\text { Chay - Oldenlandia umbellate, Dyer's Madder - } \\
\text { Rubia tinctorum }\end{array}$ & Roots & Red & Anti-Microbial, Antioxidant, Anti-Inflammatory \\
\hline $\begin{array}{l}\text { Xanthopurpurin } \\
\text { Figure } 27\end{array}$ & $\begin{array}{l}\text { Rubia tinctorum, R. Cordifolia, Bedstraw, Sweet } \\
\text { Woodruff, Indian Mulberry }\end{array}$ & $\begin{array}{l}\text { Roots, } \\
\text { Stems }\end{array}$ & Red & Anti-Microbial, Antioxidant, Anti-Inflammatory \\
\hline $\begin{array}{l}\text { Rubiadin } \\
\text { Figure } 28\end{array}$ & $\begin{array}{c}\text { Bongo - Danais fragrans, Ojnologbo - M. Longiflora, } \\
\text { Oruwo - M. Lucida, Indian Mulberry }\end{array}$ & Root Bark & Red & Anti-Microbial, Antioxidant, Anti-Inflammatory \\
\hline $\begin{array}{l}\text { Lucidin } \\
\text { Figure } 29\end{array}$ & $\begin{array}{l}\text { Shining Karamu - Coprosma lucida, Madders, Bed- } \\
\text { straws, Indian Mulberry, Relbunium Spp. }\end{array}$ & $\begin{array}{l}\text { Bark, } \\
\text { Roots }\end{array}$ & Red & Anti-Microbial, Antioxidant, Anti-Inflammatory \\
\hline $\begin{array}{c}\text { Damnacanthal } \\
\text { Figure } 30\end{array}$ & Morinda citrifolia, M. Umbellata & $\begin{array}{l}\text { Roots, } \\
\text { Stems }\end{array}$ & Red & Anti-Microbial, Antioxidant, Anti-Inflammatory \\
\hline $\begin{array}{l}\text { Munjistin } \\
\text { Figure } 31\end{array}$ & $\begin{array}{l}\text { Madders, Bedstraws, Mengkudu Hutan - Morinda } \\
\text { umbellata }\end{array}$ & $\begin{array}{l}\text { Roots, } \\
\text { Root Bark }\end{array}$ & Red & Anti-Microbial, Antioxidant, Anti-Inflammatory \\
\hline $\begin{array}{l}\text { Soranjidiol } \\
\text { Figure } 32\end{array}$ & $\begin{array}{c}\text { Suranji, Oruwo, Coprosma Spp., Indian Mulberry, } \\
\text { Rubia Yunnanensis }\end{array}$ & $\begin{array}{l}\text { Roots } \\
\text { Stems - } \\
\text { Wood, } \\
\text { Bark }\end{array}$ & Red & Anti-Microbial, Antioxidant, Anti-Inflammatory \\
\hline $\begin{array}{c}\text { Anthragallol } \\
\text { Figure } 33\end{array}$ & Madders, Shining Karamu, Indian Mulberry & $\begin{array}{l}\text { Roots, } \\
\text { Bark }\end{array}$ & Red & Anti-Microbial, Antioxidant, Anti-Inflammatory \\
\hline $\begin{array}{l}\text { Morindone } \\
\text { Figure } 34\end{array}$ & Indian Mulberry, M. Umbellate, M. Lucida & $\begin{array}{c}\text { Roots, } \\
\text { Wood, } \\
\text { Root } \\
\text { Barks, } \\
\text { Stems }\end{array}$ & Red & Anti-Microbial, Antioxidant, Anti-Inflammatory \\
\hline $\begin{array}{l}\text { Copareolatin } \\
\text { Figure } 35\end{array}$ & Coprosma Areolate & Bark & Red & Anti-Microbial, Antioxidant, Anti-Inflammatory \\
\hline - Anthraquinols & Oruwo, Cascara Sagrada, Buckthorn, Rhubarb & $\begin{array}{l}\text { Stems, } \\
\text { Wood }\end{array}$ & Red & Anti-Microbial, Antioxidant, Anti-Inflammatory \\
\hline $\begin{array}{l}\text { Oruwal, Oruwalol } \\
\text { Figure } 36\end{array}$ & Oruwo - Morinda lucida & $\begin{array}{l}\text { Stems, } \\
\text { Wood }\end{array}$ & Red & Anti-Microbial, Antioxidant, Anti-Inflammatory \\
\hline Betalains & Caryophyllales Spp. - Cacti, Amaranth, Beet & $\begin{array}{l}\text { Leaves, } \\
\text { Stems, } \\
\text { Roots }\end{array}$ & Red & Anti-Inflammatory, Antioxidant \\
\hline $\begin{array}{l}\text { Betanidin } \\
\text { Figure } 37\end{array}$ & Beta Vulgaris & $\begin{array}{l}\text { Leaves, } \\
\text { Stems, } \\
\text { Roots }\end{array}$ & Red & Anti-Inflammatory, Antioxidant \\
\hline $\begin{array}{l}\text { Betaxanthin } \\
\text { Figure } 38\end{array}$ & Swiss Chard & $\begin{array}{l}\text { Leaves, } \\
\text { Stems, } \\
\text { Roots }\end{array}$ & Red & Anti-Inflammatory, Antioxidant \\
\hline $\begin{array}{l}\text { Betacyanin } \\
\text { Figure } 39\end{array}$ & Swiss Chard & $\begin{array}{l}\text { Leaves, } \\
\text { Stems, } \\
\text { Roots }\end{array}$ & Red & Anti-Inflammatory, Antioxidant \\
\hline $\begin{array}{l}\text { Indoles - Indigotin } \\
\quad \text { Figure } 40\end{array}$ & $\begin{array}{c}\text { Indigofera tinctoria - Indigo, Woad - Isatis tinctoria, } \\
\text { Water Jasmine, Assam Indigo, Java Indigo, Dyers } \\
\text { Knotweed, Indigo Vine, Yangua }\end{array}$ & Leaves & Blue & $\begin{array}{l}\text { Anti-Microbial, Anti-Inflammatory, Anti-Fungal, } \\
\text { Analgesic, Antiproliferative, Anti-Viral }\end{array}$ \\
\hline Quinone Methides & $\begin{array}{l}\text { Rosewood, Tripterygium wilfordii, Maytenus hetero- } \\
\text { phylla, Sage }\end{array}$ & $\begin{array}{l}\text { Wood, } \\
\text { Leaves, } \\
\text { Stems }\end{array}$ & Dark Red & $\begin{array}{c}\text { Anti-Inflammatory, Antioxidant, Anti-Insect, } \\
\text { Anti-Cancer, Anti-Viral, Anti-Fungal, Anti-Bac- } \\
\text { terial }\end{array}$ \\
\hline
\end{tabular}




\begin{tabular}{|c|c|c|c|c|}
\hline $\begin{array}{l}\text { Coleone } \\
\text { Figure } 41\end{array}$ & Coleus ignarius & Leaf & Red & Anti-Septic \\
\hline $\begin{array}{l}\text { Dracorubin } \\
\text { Figure } 42\end{array}$ & Dragons Blood Plant - Dracaena Draco & Tree Resin & Red & Anti-Microbial \\
\hline $\begin{array}{l}\text { Santalin } \\
\text { Figure } 43\end{array}$ & Red Sandalwood & Wood & Red & Anti-Microbial, Antioxidant, Anti-Inflammatory \\
\hline $\begin{array}{l}\text { Phenalones - } \\
\text { Lachnanthofluo- } \\
\text { rone }\end{array}$ & Carolina Redroots, Lachnanthes tinctoria & Roots & $\begin{array}{c}\text { Red } \\
\text { Red Purple }\end{array}$ & Anti-Inflammatory, Antioxidant \\
\hline $\begin{array}{l}\text {-Curcumin } \\
\text { Figure } 44\end{array}$ & Turmeric Root & Roots & Yellow & Anti-Microbial, Antioxidant, Anti-Inflammatory \\
\hline $\begin{array}{l}\text { Pyrones - Man- } \\
\quad \text { gostin } \\
\text { Figure } 45 \& 46\end{array}$ & Garcinia mangostana - Mangosteen, Pitch Apple & Fruit & Orange Red & Antioxidant, Anti-Inflammatory \\
\hline $\begin{array}{l}\text { Thiarubrines - } \\
\text { Polyacetylenes } \\
\text { Figure } 47 \& 48\end{array}$ & Ragweed, Ambrosia chamissonis, Carrots & $\begin{array}{l}\text { Roots } \\
\text { Leaves, } \\
\text { Stems }\end{array}$ & $\begin{array}{c}\text { Red } \\
\text { Orange }\end{array}$ & $\begin{array}{l}\text { Anti-Fungal, } \\
\text { Antioxidant }\end{array}$ \\
\hline $\begin{array}{l}\text { Benzophenones } \\
\text { Figure } 49\end{array}$ & Old Fustic, Jackfruit & $\begin{array}{l}\text { Wood, } \\
\text { Twigs }\end{array}$ & Yellow & Antioxidant \\
\hline $\begin{array}{l}\text {-Maclurin \& Cy- } \\
\text { nomaclurin } \\
\text { Figure } 50\end{array}$ & $\begin{array}{l}\text { Old Fustic, Jackfruit, } \\
\text { Purple Mangosteen, } \\
\text { Mulberry Twigs }\end{array}$ & $\begin{array}{l}\text { Wood, } \\
\text { Twigs }\end{array}$ & Yellow & Antioxidant \\
\hline $\begin{array}{l}\text { Alkaloids } \\
\text { Figure } 51\end{array}$ & $\begin{array}{l}\text { Gangamau, Goldthread, Poppy Family - Papaver- } \\
\text { aceae, Ranunculaceae - Buttercups, Solanaceae - } \\
\text { Nightshades, Amaryllidaceae - Amaryllis }\end{array}$ & $\begin{array}{l}\text { Wood, } \\
\text { Bark, } \\
\text { Roots }\end{array}$ & Yellow & $\begin{array}{c}\text { Anti-Inflammatory, Analgesics, Antimicrobial, } \\
\text { Antifungal }\end{array}$ \\
\hline $\begin{array}{l}\text { Berberine } \\
\text { Figure } 52\end{array}$ & $\begin{array}{c}\text { Amur Cork Tree, Fibraurea tinctoria, Barberries, } \\
\text { Goldenseal - Hydrastis canadensis, Oregon Grape - } \\
\text { Berberis Aquifolium, Bayberry - Berberis Vulgaris, } \\
\text { Coptis - Coptis chinensis, Tree Turmeric - Berberis } \\
\text { aristata }\end{array}$ & $\begin{array}{l}\text { Wood, } \\
\text { Bark, } \\
\text { Roots }\end{array}$ & Yellow & $\begin{array}{c}\text { Anti-Inflammatory, Analgesics, Antimicrobial, } \\
\text { Antifungal }\end{array}$ \\
\hline $\begin{array}{l}\text { Canadine } \\
\text { Figure } 53\end{array}$ & $\begin{array}{l}\text { Oregon Grape, Bloodroot, Coptis Spp., Papaverace- } \\
\text { ae - Corydalis yanhusuon C. Turtschaninovii }\end{array}$ & $\begin{array}{l}\text { Wood, } \\
\text { Bark, } \\
\text { Roots }\end{array}$ & Yellow & $\begin{array}{c}\text { Anti-Inflammatory, Analgesics, Antimicrobial, } \\
\text { Antifungal }\end{array}$ \\
\hline $\begin{array}{l}\text { Phellodendrine } \\
\text { Figure } 54\end{array}$ & $\begin{array}{c}\text { Amur Cork Tree, Japanese Cork Tree - Phelloden- } \\
\text { dron Spp. }\end{array}$ & $\begin{array}{l}\text { Wood, } \\
\text { Bark, } \\
\text { Roots }\end{array}$ & Yellow & $\begin{array}{c}\text { Anti-Inflammatory, Analgesics, Antimicrobial, } \\
\text { Antifungal }\end{array}$ \\
\hline $\begin{array}{l}\text { Aporphine Alkaloid } \\
\quad \text { Figure } 55\end{array}$ & $\begin{array}{c}\text { Berberis, Mahonia Spp. - Amur Cork Tree, Japanese } \\
\text { Cork Tree, Coptis japonica }\end{array}$ & $\begin{array}{l}\text { Wood, } \\
\text { Bark, } \\
\text { Roots }\end{array}$ & Yellow & $\begin{array}{l}\text { Anti-Inflammatory, Analgesics, Antimicrobial, } \\
\text { Antifungal }\end{array}$ \\
\hline $\begin{array}{l}\text { Bisbenzylisoquino- } \\
\text { line Alkaloids } \\
\text { Figure } 56\end{array}$ & $\begin{array}{c}\text { Berberidaceae, Menispermaceae, Lauraceae, Ra- } \\
\text { nunculaceae Plant Families }\end{array}$ & $\begin{array}{l}\text { Wood, } \\
\text { Bark, } \\
\text { Roots }\end{array}$ & Yellow & $\begin{array}{c}\text { Anti-Inflammatory, Analgesics, Antimicrobial, } \\
\text { Antifungal }\end{array}$ \\
\hline $\begin{array}{l}\text { Benzophenanthri- } \\
\text { dine Alkaloids } \\
\text { Figure } 57\end{array}$ & Bloodroot & $\begin{array}{l}\text { Wood, } \\
\text { Bark, } \\
\text { Roots }\end{array}$ & Yellow & $\begin{array}{c}\text { Anti-Inflammatory, Analgesics, Antimicrobial, } \\
\text { Antifungal }\end{array}$ \\
\hline $\begin{array}{l}\text { Indoloquinoline } \\
\text { Alkaloids } \\
\text { Figure } 58\end{array}$ & Cryptolepis sanguinolenta & $\begin{array}{l}\text { Wood, } \\
\text { Bark, } \\
\text { Roots }\end{array}$ & Yellow & $\begin{array}{c}\text { Anti-Inflammatory, Analgesics, Antimicrobial, } \\
\text { Antifungal }\end{array}$ \\
\hline $\begin{array}{l}\text { Tannins } \\
\text { Figure } 59\end{array}$ & $\begin{array}{l}\text { Sessile Oak, Aleppo Oak, Sticky Alder, Western } \\
\text { Hemlock, Sicilian Sumac, Staghorn, Coronillo, } \\
\text { Myrobalans, Bakli, Red Mangrove, Divi-Divi Cutch } \\
\text { Tree, Areca Plam, Pomegranate }\end{array}$ & $\begin{array}{l}\text { Wood, } \\
\text { Bark, } \\
\text { Leaves, } \\
\text { Acorns, } \\
\text { Galls }\end{array}$ & $\begin{array}{l}\text { Brown, } \\
\text { Black, } \\
\text { Red }\end{array}$ & Anti-Inflammatory, Antioxidant \\
\hline
\end{tabular}




\begin{tabular}{|c|c|c|c|c|}
\hline $\begin{array}{l}\text {-Gallotannins } \\
\text { Figure } 60\end{array}$ & $\begin{array}{l}\text { Quercus infectoria, Rhus javanica, Cotinus coggygria, } \\
\text { Terminalia chebula, Aleppo Galls, Sicilian Sumac, } \\
\text { Smooth Sumac, Fragrant Sumac }\end{array}$ & $\begin{array}{l}\text { Wood, } \\
\text { Bark, } \\
\text { Leaves, } \\
\text { Acorns }\end{array}$ & $\begin{array}{c}\text { Brown, } \\
\text { Black, } \\
\text { Red }\end{array}$ & Anti-Inflammatory, Antioxidant \\
\hline $\begin{array}{l}\text { Tara Tannin } \\
\text { Figure } 61\end{array}$ & $\begin{array}{c}\text { Caesalpinia spinosa, } \\
\text { Divi Divi, Tara } \\
\text { Holdback }\end{array}$ & $\begin{array}{l}\text { Wood, } \\
\text { Bark, } \\
\text { Leaves, } \\
\text { Acorns }\end{array}$ & $\begin{array}{l}\text { Brown, } \\
\text { Black, } \\
\text { Red }\end{array}$ & $\begin{array}{l}\text { Anti-Inflammatory, } \\
\text { Antioxidant }\end{array}$ \\
\hline $\begin{array}{l}\text {-Ellagitannins } \\
\text { Figure } 62\end{array}$ & $\begin{array}{l}\text { Durmast, Sessile Oak, Aleppo Galls, Levant Galls, } \\
\text { Walloon Oak }\end{array}$ & $\begin{array}{l}\text { Wood, } \\
\text { Bark, } \\
\text { Leaves, } \\
\text { Acorns, } \\
\text { Galls }\end{array}$ & $\begin{array}{c}\text { Brown, } \\
\text { Black, } \\
\text { Red }\end{array}$ & Anti-Inflammatory, Antioxidant \\
\hline $\begin{array}{l}\text { Ellagic Acid } \\
\text { Figure } 63\end{array}$ & $\begin{array}{l}\text { Quebracho, Indian Sumac, Cangara, Ngalama, } \\
\text { Pomegranates, Pecans, Walnuts }\end{array}$ & $\begin{array}{l}\text { Wood, } \\
\text { Bark, } \\
\text { Leaves, } \\
\text { Acorns }\end{array}$ & $\begin{array}{l}\text { Brown, } \\
\text { Black, } \\
\text { Red }\end{array}$ & Anti-Inflammatory, Antioxidant \\
\hline $\begin{array}{l}\text { Corilagin } \\
\text { Figure } 64\end{array}$ & $\begin{array}{l}\text { Rhus javanica, Anogeissus latifolia, Divi-Divi, Myro- } \\
\text { balan, Indian Almond }\end{array}$ & $\begin{array}{l}\text { Leaves, } \\
\text { Fruits, } \\
\text { Pods }\end{array}$ & $\begin{array}{c}\text { Brown, } \\
\text { Black, } \\
\text { Red }\end{array}$ & Anti-Inflammatory, Antioxidant \\
\hline $\begin{array}{l}\text { Chebulinic Acid } \\
\text { Figure } 65\end{array}$ & $\begin{array}{l}\text { Terminalia chebula, Chebulic myrobalan, Beleric } \\
\text { myrobalan }\end{array}$ & Fruits & $\begin{array}{c}\text { Brown, } \\
\text { Black, } \\
\text { Red }\end{array}$ & Anti-Inflammatory, Antioxidant \\
\hline $\begin{array}{l}\text { Tellimagrandin I } \\
\text { Figure } 66\end{array}$ & $\begin{array}{l}\text { Woodfordia fruticose, Q. Robur, Q. Petraea, Rosa } \\
\text { Rugosa, Walnut }\end{array}$ & $\begin{array}{l}\text { Bark, } \\
\text { Galls }\end{array}$ & $\begin{array}{c}\text { Brown, } \\
\text { Black, } \\
\text { Red }\end{array}$ & Anti-Inflammatory, Antioxidant \\
\hline $\begin{array}{l}\text { Vescalin And Casta- } \\
\text { lin Figure } 67 \& 68\end{array}$ & $\begin{array}{c}\text { Sweet Chestnuts - Castanea, Oaks - Quercus Spp. - } \\
\text { Durmast, Sessile Oak }\end{array}$ & $\begin{array}{l}\text { Wood, } \\
\text { Bark, } \\
\text { Leaves, } \\
\text { Acorns }\end{array}$ & $\begin{array}{c}\text { Brown, } \\
\text { Black, } \\
\text { Red }\end{array}$ & Anti-Inflammatory, Antioxidant \\
\hline $\begin{array}{l}\text { Vescalagin and } \\
\text { Castalagin } \\
\text { Figure } 69 \& 70\end{array}$ & Chestnuts - Castanea, Oaks - Quercus Spp. & $\begin{array}{l}\text { Wood, } \\
\text { Bark, } \\
\text { Leaves, } \\
\text { Acorns }\end{array}$ & $\begin{array}{c}\text { Brown, } \\
\text { Black, } \\
\text { Red }\end{array}$ & Anti-Inflammatory, Antioxidant \\
\hline $\begin{array}{l}\text { Roburins A, B, C, D } \\
\text { Figure } 71,72 \& 73\end{array}$ & $\begin{array}{c}\text { Pedunculate Oak - Q. Robur, Sessils Oak - } Q . \\
\text { Petraea }\end{array}$ & $\begin{array}{l}\text { Wood, } \\
\text { Bark, } \\
\text { Leaves, } \\
\text { Acorns }\end{array}$ & $\begin{array}{c}\text { Brown, } \\
\text { Black, } \\
\text { Red }\end{array}$ & Anti-Inflammatory, Antioxidant \\
\hline $\begin{array}{l}\text { Alnusiin } \\
\text { Figure } 74\end{array}$ & Alnus sieboldiana, Grey Alder, Oregon Alder & $\begin{array}{l}\text { Wood, } \\
\text { Bark, } \\
\text { Leaves, } \\
\text { Acorns }\end{array}$ & $\begin{array}{c}\text { Brown, } \\
\text { Black, } \\
\text { Red }\end{array}$ & Anti-Inflammatory, Antioxidant \\
\hline $\begin{array}{l}\text { Punicalin } \\
\text { Figure } 75\end{array}$ & $\begin{array}{c}\text { Punica granatum, } \\
\text { Cangara - Combretum glutinosum }\end{array}$ & $\begin{array}{c}\text { Rind, } \\
\text { Roots, } \\
\text { Trunk, } \\
\text { Branches }\end{array}$ & $\begin{array}{c}\text { Brown, } \\
\text { Black, } \\
\text { Red }\end{array}$ & Anti-Inflammatory, Antioxidant \\
\hline $\begin{array}{c}\text { Granatins A, B } \\
\text { Figure } 76\end{array}$ & Terminalia chebula, Pomegranate & Fruits & $\begin{array}{l}\text { Brown, } \\
\text { Black, } \\
\text { Red }\end{array}$ & Anti-Inflammatory, Antioxidant \\
\hline $\begin{array}{l}\text { Woodfruticosin } \\
\text { Figure } 77\end{array}$ & Dhawai, Woodfordia fruticose & Bark & $\begin{array}{c}\text { Brown, } \\
\text { Black, } \\
\text { Red }\end{array}$ & Anti-Inflammatory, Antioxidant \\
\hline $\begin{array}{l}\text {-Proanthocyanidins } \\
\text { Figure } 78\end{array}$ & $\begin{array}{c}\text { Grey Alder, Red Alder, Western Hemlock, Tizra, } \\
\text { Gambier Bush }\end{array}$ & $\begin{array}{l}\text { Wood, } \\
\text { Bark, } \\
\text { Leaves, } \\
\text { Acorns }\end{array}$ & $\begin{array}{l}\text { Brown, } \\
\text { Black, } \\
\text { Red }\end{array}$ & Anti-Inflammatory, Antioxidant \\
\hline $\begin{array}{c}\text { Flavan } \\
\text { Figure } 79\end{array}$ & $\begin{array}{c}\text { Mastic Tree, Cyprus turpentine, Sicilian sumac, } \\
\text { Quebracho, Cutch Tree }\end{array}$ & $\begin{array}{l}\text { Wood, } \\
\text { Bark, } \\
\text { Leaves, } \\
\text { Acorns }\end{array}$ & $\begin{array}{c}\text { Brown, } \\
\text { Black, } \\
\text { Red }\end{array}$ & Anti-Inflammatory, Antioxidant \\
\hline
\end{tabular}




\begin{tabular}{|c|c|c|c|c|}
\hline Propelargonidins & $\begin{array}{c}\text { Gambier - Uncaria gambir, Western Hemlock, } \\
\text { Gambier Bush, Areca Plam }\end{array}$ & Leaves & $\begin{array}{l}\text { Brown, } \\
\text { Black, } \\
\text { Red }\end{array}$ & Anti-Inflammatory, Antioxidant \\
\hline $\begin{array}{c}\text { Procyanidins } \\
\text { Figure } 80\end{array}$ & $\begin{array}{l}\text { Oaks, Pines - Pinus halepensis, Spruce, Hemlock, } \\
\text { Larch, Willows - Salix viminalis, Apple, Mangrove - } \\
\text { Ceriops tagal, California Pepper Tree }\end{array}$ & Barks & $\begin{array}{l}\text { Brown, } \\
\text { Black, } \\
\text { Red }\end{array}$ & Anti-Inflammatory, Antioxidant \\
\hline $\begin{array}{l}\text { Tetramers } \\
\text { Figure } 81\end{array}$ & $\begin{array}{c}\text { Dye Yam, Wattles, Oak, Quebracho, Pine, Birch, } \\
\text { Willow, Eucalyptus }\end{array}$ & $\begin{array}{l}\text { Wood, } \\
\text { Bark, } \\
\text { Leaves, } \\
\text { Acorns }\end{array}$ & $\begin{array}{l}\text { Brown, } \\
\text { Black, } \\
\text { Red }\end{array}$ & Anti-Inflammatory, Antioxidant \\
\hline $\begin{array}{l}\text { Profisetinidins } \\
\text { Figure } 82\end{array}$ & $\begin{array}{l}\text { Wattles - Acacia mearnsii, Quebrachos - schinopsis } \\
\text { Spp., Babla, Pines, Oaks, Alders, Eucalyptus }\end{array}$ & $\begin{array}{l}\text { Heart- } \\
\text { wood, } \\
\text { Pods, } \\
\text { Bark }\end{array}$ & $\begin{array}{l}\text { Brown, } \\
\text { Black, } \\
\text { Red }\end{array}$ & Anti-Inflammatory, Antioxidant \\
\hline Prorobinetinidins & Wattles & $\begin{array}{l}\text { Heart- } \\
\text { wood }\end{array}$ & $\begin{array}{l}\text { Brown, } \\
\text { Black, } \\
\text { Red }\end{array}$ & Anti-Inflammatory, Antioxidant \\
\hline Phlobatannins & $\begin{array}{c}\text { Western Hemlock, Tengar, Red Mangrove, Acacia } \\
\text { Nilotica }\end{array}$ & $\begin{array}{l}\text { Wood, } \\
\text { Bark, } \\
\text { Leaves, } \\
\text { Acorns }\end{array}$ & $\begin{array}{l}\text { Brown, } \\
\text { Black, } \\
\text { Red }\end{array}$ & Anti-Inflammatory, Antioxidant \\
\hline - Complex Tannins & $\begin{array}{c}\text { Sweet Chestnuts, } \\
\text { Psidium guajava, Oaks }\end{array}$ & $\begin{array}{l}\text { Wood, } \\
\text { Bark, } \\
\text { Leaves, } \\
\text { Acorns }\end{array}$ & $\begin{array}{l}\text { Brown, } \\
\text { Black, } \\
\text { Red }\end{array}$ & Anti-Inflammatory, Antioxidant \\
\hline $\begin{array}{l}\text { Acutissimin A, B } \\
\text { Figure } 83 \text { \& } 84\end{array}$ & $\begin{array}{c}\text { Oaks - Kunigi - Quercus acutissima, Durmast, } \\
\text { Sessile Oak, Urajirogashi - Q. Stenophylla, Chestnuts } \\
\text { - Kuri - Castanea Crenata }\end{array}$ & $\begin{array}{l}\text { Wood, } \\
\text { Bark, } \\
\text { Leaves, } \\
\text { Acorns }\end{array}$ & $\begin{array}{c}\text { Brown, } \\
\text { Black, } \\
\text { Red }\end{array}$ & Anti-Inflammatory, Antioxidant \\
\hline Anogeissusins A, B & Anogeissus acuminata & Bark & $\begin{array}{c}\text { Brown, } \\
\text { Black, } \\
\text { Red }\end{array}$ & Anti-Inflammatory, Antioxidant \\
\hline
\end{tabular}<smiles>O=C1c2ccccc2C(=O)c2ccccc21</smiles>

Figure 12: Anthraquinone<smiles>Cc1cc(O)c2c(c1)C(=O)c1cccc(O)c1C2=O</smiles>

Figure 14: Chrysophanol<smiles>O=C1c2cc(CO)cc(O)c2C(=O)c2c(Cl)cc(Cl)cc21</smiles>

Figure 15: Aloe-emodin

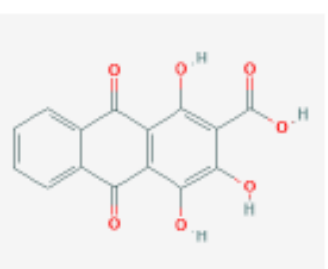

Figure 13: Pseudopurpurin [16]<smiles>O=C(O)c1cc(O)c2c(c1)C(=O)c1cccc(O)c1C2=O</smiles>

Figure 16: Rhein 


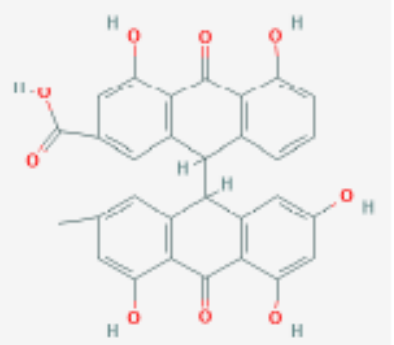

Figure 17: Rheidin A [16]

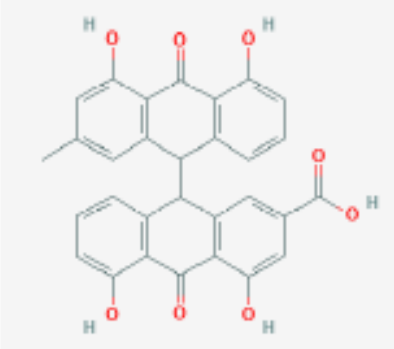

Figure 18: Rheidin B [16]

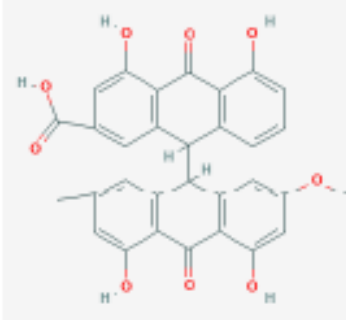

Figure 19: Rheidin C [16]<smiles>Cc1cc(O)c2c(c1)C(=O)c1cc(O)cc(O)c1C2=O</smiles>

Figure 20: Emodin<smiles>Cc1cc(O)c2c(c1O)C(=O)c1cccc(O)c1C2=O</smiles>

Figure 23: Islandicin<smiles>O=C1c2ccccc2C(=O)c2c1ccc(O)c2O</smiles>

Figure 26: Alizarin<smiles>COc1cc(O)c2c(c1)C(=O)c1cc(C)cc(O)c1C2=O</smiles>

Figure 21: Physcion<smiles>COc1cc(O)c2c(c1O)C(=O)c1cc(C)cc(O)c1C2=O</smiles>

Figure 24: Xanthorin<smiles>O=C1c2ccccc2C(=O)c2c(O)cc(O)cc21</smiles>

Figure 27: Xanthopurpurin

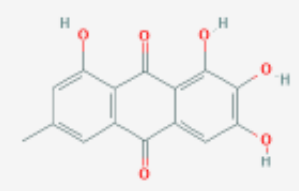

Figure 22: Alaternin [16]

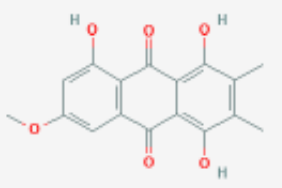

Figure 25: Ventinones A [16]<smiles>Cc1c(O)cc2c(c1O)C(=O)c1ccccc1C2=O</smiles>

Figure 28: Rubiadin 


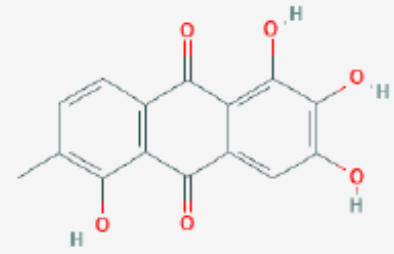

Figure 35: Copareolatin [16]

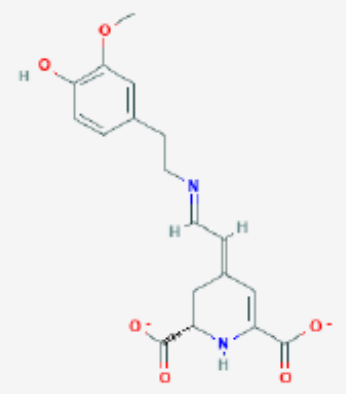

Figure 38: Betaxanthin [16]

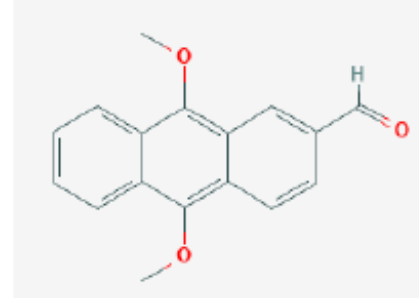

Figure 36: Oruwal [16]

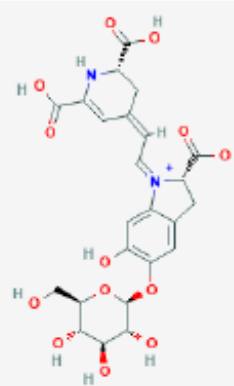

Figure 39: Betacyanin [16]

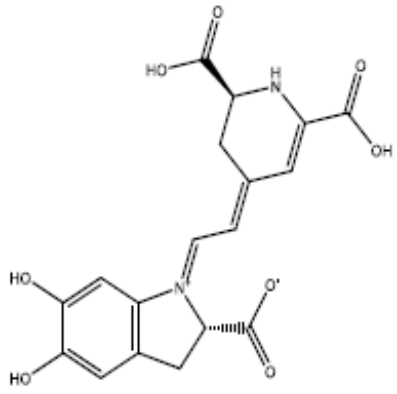

Figure 37: Betanidin

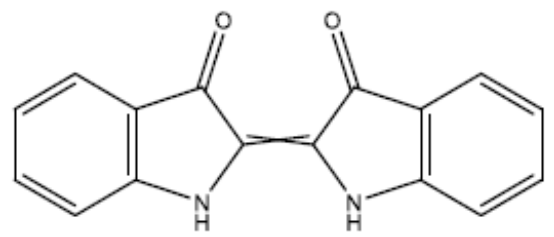

Figure 40: Indigotin

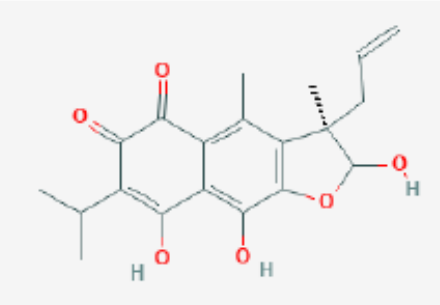

Figure 41: Coleone [16]<smiles>COc1cc2oc3c(C)c(=O)cc4oc(-c5ccccc5)cc4c3c2c2c1CCC(c1ccccc1)O2</smiles>

Figure 42: Dracorubin<smiles>COc1c(O)cc2c(c1O)C(=O)C1=C(CCCC1)C2=O</smiles>

Figure 43: Santalin<smiles>COc1cc(/C=C/C(=O)CC(=O)/C=C/c2ccc(O)c(OC)c2)ccc1O</smiles>

Figure 44: Curcumin

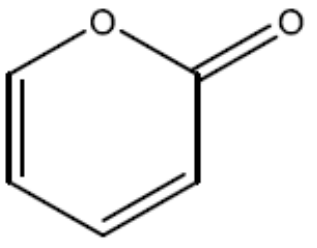

Figure 45: Pyrones 
<smiles>COc1c(O)cc2oc3cc(O)c(CC=C(C)C)c(O)c3c(=O)c2c1CC=C(C)C</smiles>

Figure 46: Mangostin

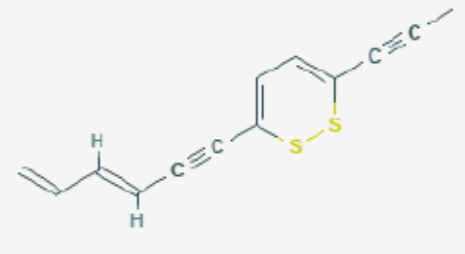

Figure 47: Thiarubrine [16]
$\mathrm{H}$<smiles>C1#CC#C1</smiles>

Figure 48: Polyacetylene [16]<smiles>O=C(c1ccccc1)c1ccccc1</smiles>

Figure 49: Benzophenones<smiles>O=C(c1ccc(O)c(O)c1)c1c(O)cc(O)cc1O</smiles>

Figure 50: Maclurin

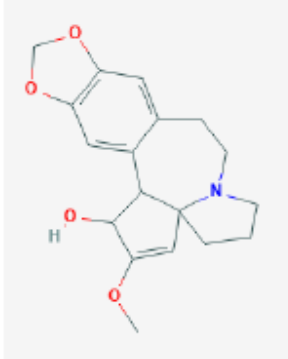

Figure 51: Alkaloid<smiles>COc1ccc2cc3[n+](cc2c1OC)CCc1cc2c(cc1-3)OCO2</smiles>

Figure 52: Berberine

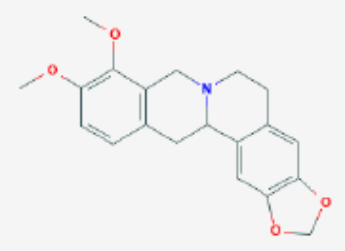

Figure 53: Canadine [16]<smiles>COc1cc2c(cc1O)[C@]1(C)Cc3cc(OC)c(O)cc3[N+]1(C)CC2</smiles>

Figure 54: Phellodendrine<smiles>CN1CCc2cccc3c2[C@]1(C)Cc1ccccc1-3</smiles><smiles>c1ccc(Cc2cc3ccccc3c(Cc3ccccc3)n2)cc1</smiles><smiles>c1ccc2c(c1)ccc1ncc3ccccc3c12</smiles>

Figure 55: Aporphine

57: Benzophenanthridine 


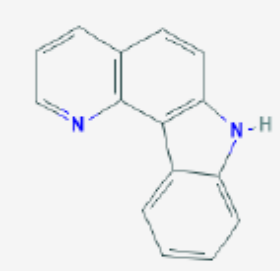

Figure 58: Indoloquinoline [16]<smiles>O=C(OCC1OC(OC(=O)c2cc(O)c(O)c(O)c2)C(O)C(OC(=O)c2cc(O)c(O)c(O)c2)C1O)c1cc(O)c(O)c(O)c1</smiles>

Figure 59: Tannin<smiles>O=C(OC[C@H]1O[C@@H](OC(=O)c2cc(O)c(O)c(OC(=O)c3cc(O)c(O)c(O)c3)c2)[C@H](OC(=O)c2cc(O)c(O)c(OC(=O)c3cc(O)c(O)c(O)c3)c2)[C@H](OC(=O)c2cc(O)c(O)c(OC(=O)c3cc(O)c(O)c(O)c3)c2)[C@@H]1OC(=O)c1cc(O)c(O)c(OC(=O)c2cc(O)c(O)c(O)c2)c1)c1cc(O)c(O)c(O)c1</smiles>

Figure 60: Gallotannin

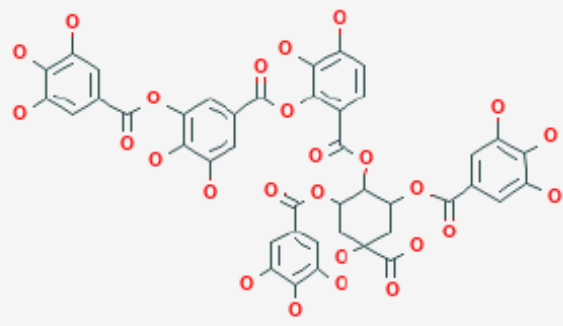

Figure 61: Tara tannin [16]

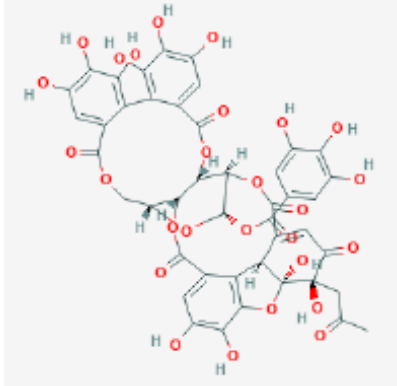

Figure 62: Ellagitannin [16]<smiles>O=c1oc2c(O)c(O)cc3c(=O)oc4c(O)c(O)cc1c4c23</smiles>

Figure 63: Ellagic acid 
<smiles>O=C(OC[C@H]1O[C@@H](OC(=O)c2cc(O)c(O)c(O)c2)[C@H](O)[C@H](OC(=O)c2cc(O)c(O)c(O)c2O)[C@@H]1O)c1cc(O)c(O)c(O)c1</smiles>

Figure 64: Corilagin

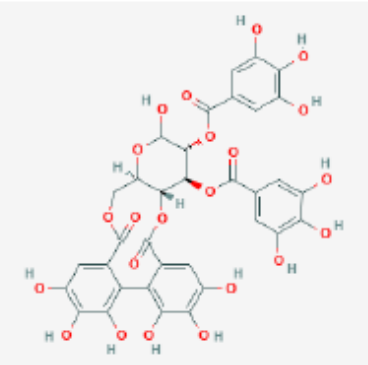

Figure 66: Tellimagrandin I [16]

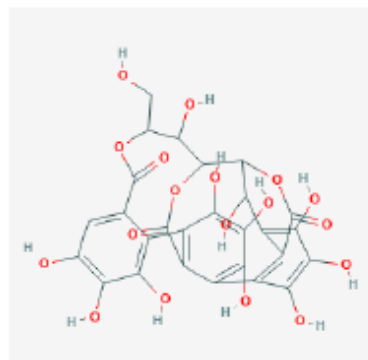

Figure 67: Vescalin [16]

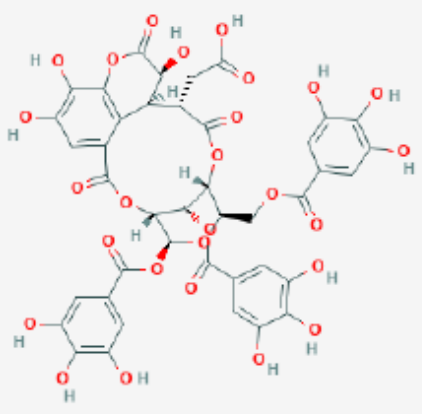

Figure 65: Chebulinic acid

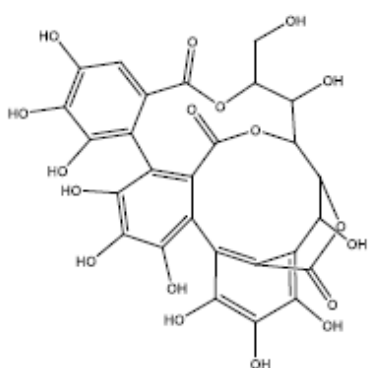

Figure 68: Castalin

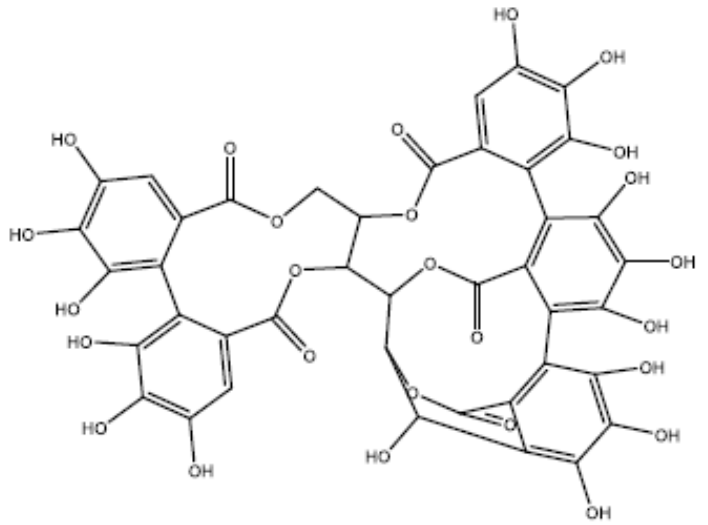

Figure 69: Vescalagin

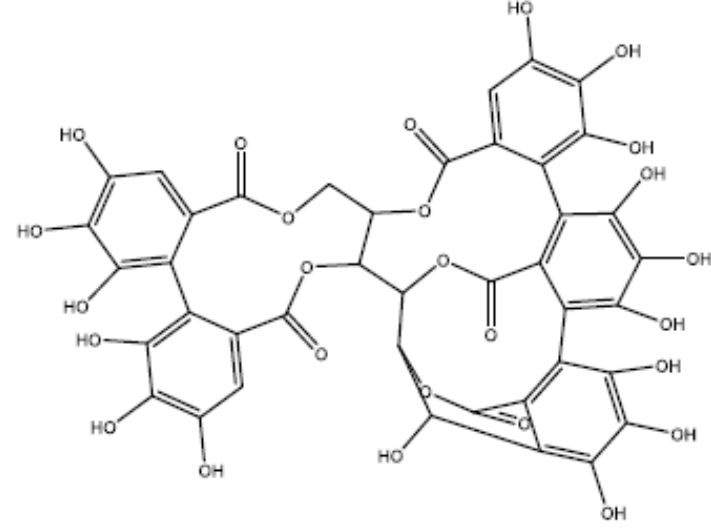

Figure 70: Castalagin

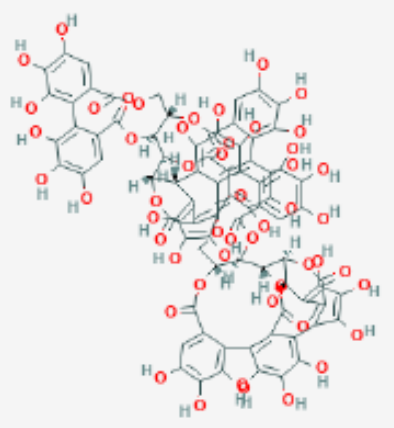

Figure 71: Roburin A [16]

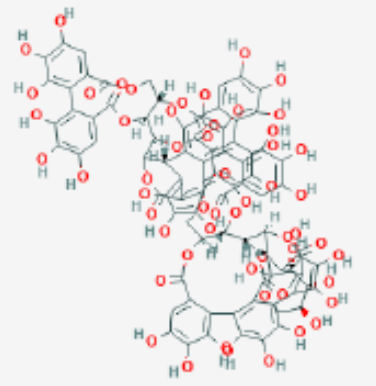

Figure 72: Roburin B [16]

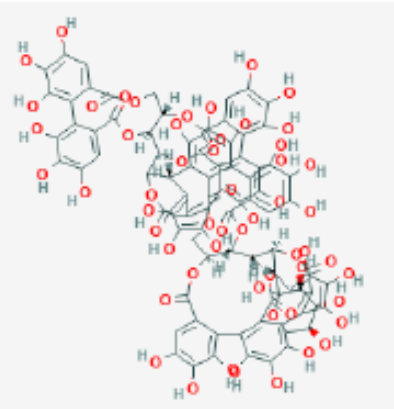

Figure 73: Roburin C [16] 


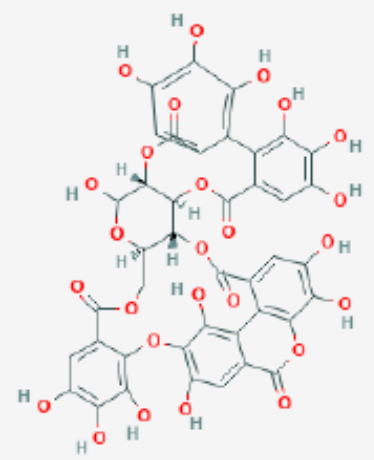

Figure 74: Alnusiin [16]

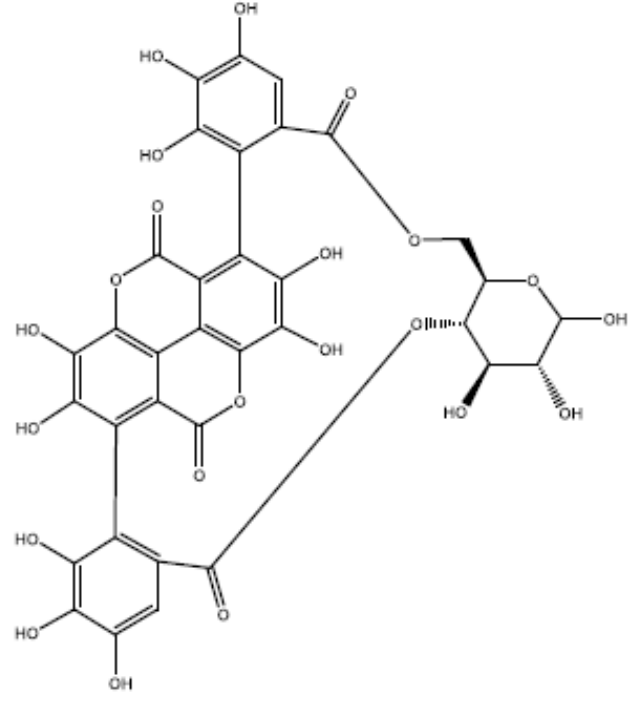

Figure 75: Punicalin

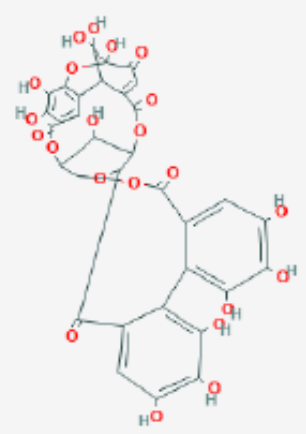

Figure 76: Granatins A [16]

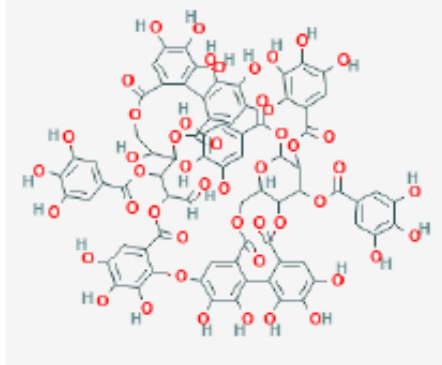

Figure 77: Woodfruticosin [16]

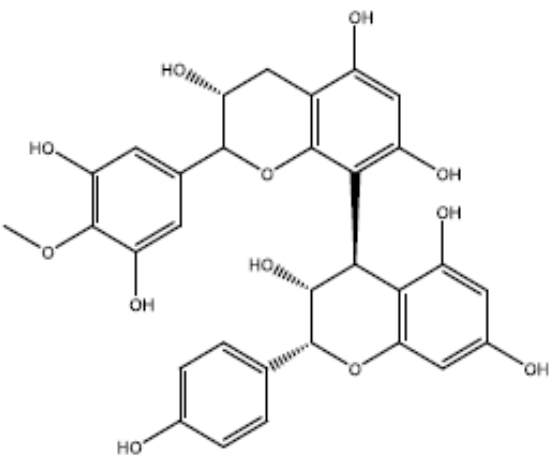

Figure 78: Proanthocyanidin<smiles>c1ccc(C2CCc3ccccc3O2)cc1</smiles>

Figure 79: Flavan

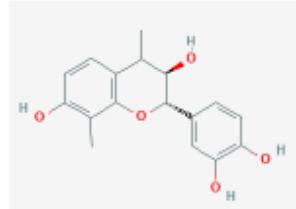

Figure 82: Profisetinidin<smiles>Cc1cc(C)c2c(c1)OC(c1ccc(O)c(O)c1)C(CCC1(c3ccc(C)c(O)c3)Oc3cc(I)cc(C)c3C(C)C1C)C2</smiles>

Figure 80: Procyanidin

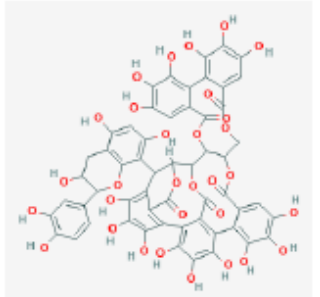

Figure 83: Acutissimin A [16] Figure 84: Acutissimin B [16] 
The academic literature on the chemical structures of bioactive components contained in biological pigments is accentuated herein. The naturally occurring therapeutic constituents adhere to the microfibrillar fabric structure and impart healing to the wearer [18]. For example, the treatment of cotton fabric with neem leaves extract has revealed the anti-microbial efficacy of the treated fabric against Staphylococcus aureus and Escherichia coli strains of bacteria. The anti-microbial activity is attributed to the presence of the bioactive component azadirachtin (C35H44016) intrinsically contained in neem leaves [19]. Similarly, previous research has established a 50:50 combination of wild turmeric and holy basil concoction application on knitted cotton fabrics to be effective against bacterial strains. The bioactive component being germacrene (C15H22O) and eugenol (C10H12O2) inherently present in turmeric and basil, respectively [20]. In the same vein, the researcher has attempted to evaluate the Ultraviolet Protection factor of cotton fabric treated with green, red, and black tea extracts. The results of this investigation show that UPF was excellent for cotton fabrics treated with green and black tea [21]. There remain several aspects of sustainability and clinical trials overlooked in the aforesaid studies. Considering all this evidence a holistic multidisciplinary research approach at international-individual stratum is necessary for global thriveability.

\section{Functional Applications of Plant-Based Materials on Textiles Substrates}

Herein the most recent, and innovative examples of plantbased biomaterial research studies for functional and ecological textiles are detailed. Table 3 classifies various herbal plants and their prospective application on natural textile substrates for functionality and coloration.

Table 3: Application of herbs on fabrics for coloration and functionality [22].

\begin{tabular}{|c|c|c|c|c|}
\hline Natural Dye & Chemical Pigment & Image & Colour & Medicinal Benefits \\
\hline Turmeric & Curcumin & & Yellow & Antioxidant, Anti-Septic and Anti-Viral \\
\hline Saffron & $\begin{array}{l}\text { Crocin, Crocetin, Picrocrocin, } \\
\text { Safranal }\end{array}$ & & $\begin{array}{l}\text { Golden } \\
\text { Yellow }\end{array}$ & $\begin{array}{l}\text { Anti-Inflammatory, Anti-Depressant, } \\
\text { Gingival Sedative }\end{array}$ \\
\hline Safflower & Carthamin & & Red & Rheumatism, Cytotoxic, Anti-Platelet \\
\hline Annato & $\begin{array}{l}\text { Carotenoids - } \\
\text { Bixin, Norbixin }\end{array}$ & & Reddish Orange & Astringent, Anti-Microbial, Antioxidant \\
\hline Pomegranate & $\begin{array}{c}\text { Anthocyanins - Punicalagin, } \\
\text { Punicalin }\end{array}$ & & Red & $\begin{array}{l}\text { Anti-Carcinogenic, Anti-Viral, Anti-Ath- } \\
\text { erosclerotic }\end{array}$ \\
\hline Tomato & $\begin{array}{c}\text { Carotenoids - Lycopene, } \\
\text { Lutein }\end{array}$ & & Yellow & Antioxidant, Vitamin-A \\
\hline
\end{tabular}




\begin{tabular}{|c|c|c|c|c|}
\hline \multirow{2}{*}{ Paprika } & $\begin{array}{c}\text { Carotenoids - Capsanthin, } \\
\text { Capsorubin }\end{array}$ & Red & Rheumatism, Lumbago, Neuralgia \\
\hline Tagetus & Lutein & Yellow to Orange & Aromatic, Sores, Ulcers, Eczema, Rheu- \\
matism
\end{tabular}

Anti-microbial fabrics, for a healthy lifestyle, our day to day interaction with textiles demand extra protection from a microorganism that we encounter in our daily life. For wound infection control an eco-smart textile biomaterial was investigated by Kole and colleagues wherein, the cotton fabric was treated with nano emulsion of anti-sceptic, and anti-microbial tetrahydroxy Curcumin. Response surface design and regression statistical analysis were performed. The textile material was treated with 0.5 $\%$ of optimized nano emulsion with nanoparticle size being 100 $300 \mathrm{~nm}$, zeta potential -30.1 to $31.1 \mathrm{mV}$, and medicinal content 80 $83.3 \%$ [23]. Cyclodextrin and polycarboxylic acid were employed as crosslinking agents applied by the exhaust method and optimized using the surface response method. The treated fabric was tested for anti-microbial efficiency against gram-positive and gramnegative bacteria.
"In this research, an empirical relationship was established and demonstrated by a secondary-order polynomial equation based on results obtained. Analysis of variance, ANOVA, showed a high coefficient of determination value $\mathrm{R}^{2}=98.41$ for gram-positive bacteria and $\mathrm{R}^{2}=96.36$ for gram-negative bacteria as compared with standard erythromycin strip. The 3D counter graphs reveal that amongst the selected variables, the squared effect of concentration, temperature, and time for both gram-positive bacteria B. Subtilis and gram-negative bacteria $E$. Coli shows the largest effect on zone inhibition, refer to Figure 85 [22]. It was concluded that the response surface methodology was the most suitable method to optimize the anti-microbial properties and maximize the zone of inhibition". The above study performs a clear and valuable statistical analysis with the latest tools. However, the paper does not mention on environmental benefits of the 'eco-friendly' approach of research undertaken (Figure 86).

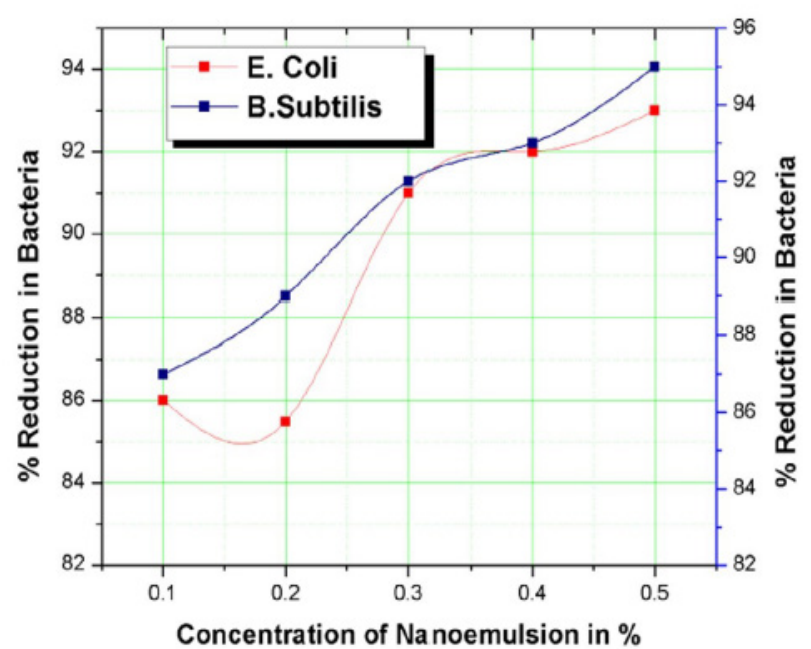

Figure 85: Gram-positive bacteria (B. Subtilis) and gram-negative bacteria (E. Coli) growth reduction graph [23]. 

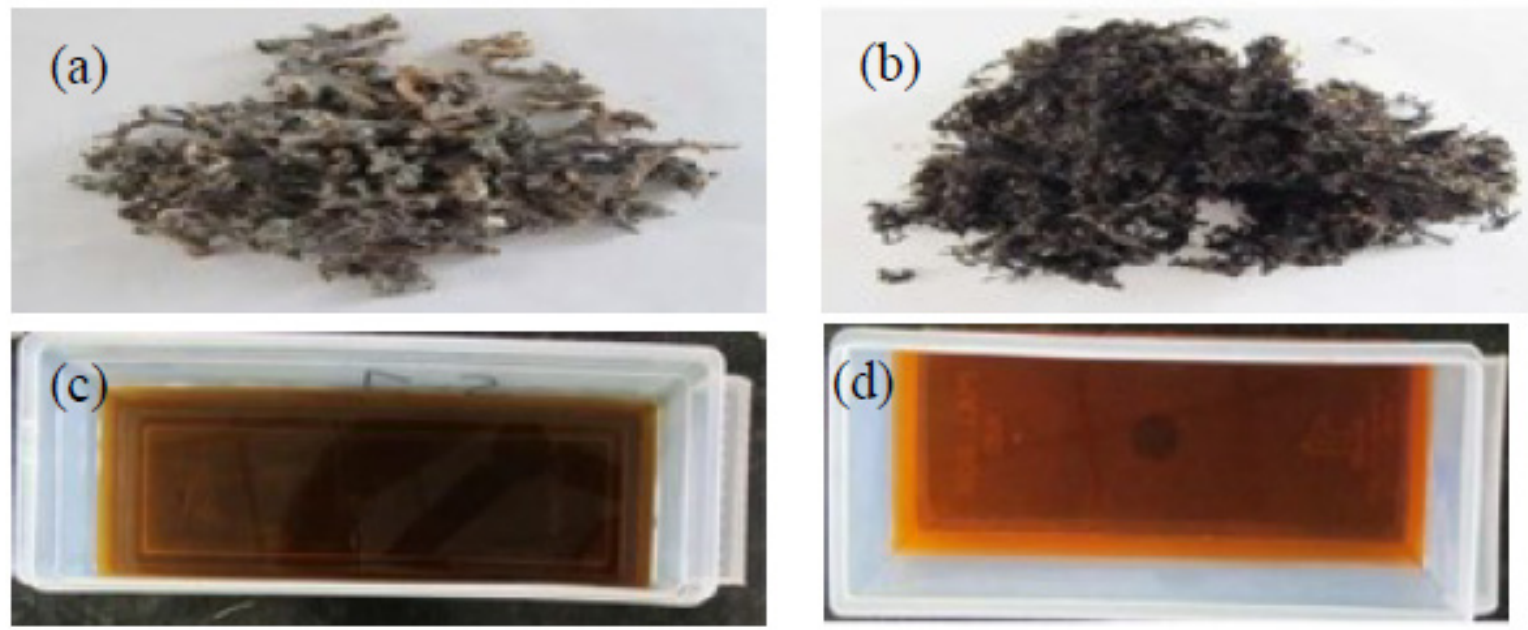

Figure 86: Red seaweed partly crushed (Above) and Red seaweed extract solution (Below) [24].

Facial masks, likewise, Janarthanan and Kumar implemented Red seaweed plant extracts namely Kappaphycus alvarezii and Acanthophora spicifera for making the textile mask for anti-aging treatment, refer to Figure 86. The antioxidant and anti-aging bioactive components present in the red seaweeds were identified with Fourier Transform infrared spectroscopy (FTIR). The fibroblast collagen layers impregnated with red seaweeds were assessed with the Scanning Electron Microscope (SEM). The FTIR revealed the presence of bio-active compounds namely, alkaloids, carotenoids, flavonoids, terpenoids, phlorotannins, and saponins, refer to Table 4. The antioxidant property evaluated with UV - Spectroscopy increased with an increase in the concentration of red seaweed [24].

Table 4: The functional groups present on the red seaweed treated cotton fabric [24].

\begin{tabular}{|c|c|}
\hline Bonds & Functional groups \\
\hline $\mathrm{N}-\mathrm{H}$ & Amines \\
\hline $\mathrm{C}-\mathrm{H}$ & Aldehydes \\
\hline $\mathrm{O}-\mathrm{H}$ & Carboxylic acids \\
\hline $\mathrm{C}-\mathrm{C}$ & Ketones \\
\hline $\mathrm{P}-\mathrm{H}$ & Phosphines \\
\hline $\mathrm{C}=\mathrm{C}$ & Terpenes \\
\hline $\mathrm{C}-\mathrm{H}$ & Alkanes \\
\hline $\mathrm{S}=\mathrm{O}$ & Sulfonates \\
\hline
\end{tabular}

The wicking ability, air, and water vapor permeability, of the red seaweed treated fabrics, was lower than the untreated fabrics, refer to Table 5. The in vitro cell morphology of cultured fibroblast collagen with red seaweed extract with SEM clearly showed that there were high surface smoothness and cell aggregation of $95 \%$ with $K$. Alvarezii and $70 \%$ with A. Spicifera. Thus, facilitating skin tightening, anti-sagging, and wrinkle-smoothing properties. The study suggests its future applications for making wound dressing, surgical gowns, face mask, gauze materials, bandages, and compression garments [24]. However, the above study excludes anti-microbial investigations as performed in the study which could further support the functionality theory in research.

Table 5: Red seaweed extract antioxidant property on cotton fabric samples [24].

\begin{tabular}{|c|c|c|}
\hline Concentration $(\mathrm{g} / \mathrm{ml})$ & \multicolumn{2}{|c|}{ DPPH free radical scavenging activity \% inhibition } \\
\hline $5 \times 10^{-5}$ & 45 & 29 \\
\hline $10 \times 10^{-4}$ & 52 & 32 \\
\hline $15 \times 10^{-4}$ & 61 & 33 \\
\hline $20 \times 10^{-4}$ & 70 & 34 \\
\hline $25 \times 10^{-4}$ & 77 & 39 \\
\hline
\end{tabular}


Solar cell activation, moreover, the natural dyes from red amaranth leaves were extracted which yield green color in ethanol extraction and red-violet color in distilled water extraction. Indicating the presence of betalain and chlorophyll pigments, respectively. They exhibited good photosensitizing properties for solar cell activation and textiles applications [25]. There is a dearth of collaborating literature on the held theory.
Biosensors, innovatively a universal $\mathrm{pH}$ sensor biocomposite has been developed by Devarayan and Suhk from the natural pigment of red cabbage on nonwoven cellulosic nanofibers. The biosensor detects $\mathrm{pH}$ 1-14 exhibiting distinct color at each $\mathrm{pH}$. This halochromic changeover is reversible. The biosensor is recyclable, stable to temperature and time. It is expected to be utilized for wellbeing monitoring purposes, refer to Figure 87 [26].

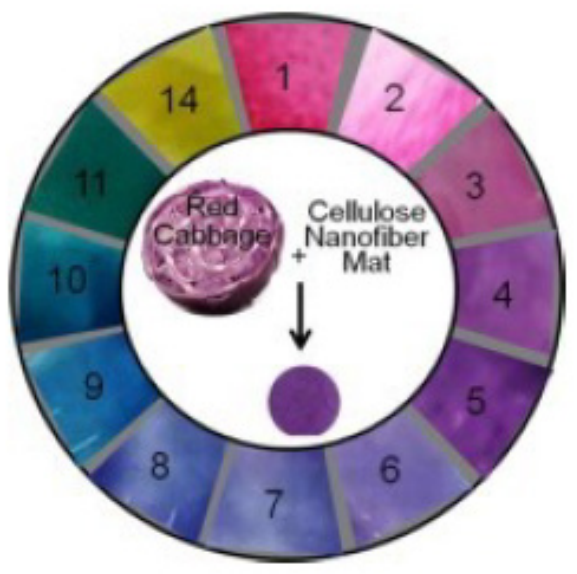

Figure 87: pH biosensor from Red-cabbage pigment on non-woven cellulosic nanofibers [26].

Beetblue, the natural colors are safe to human health, and the environment therefore increasingly preferred for food, cosmetics, textile, and drugs endues. However, itis difficult to harness thenatural blue pigment from animals and microorganisms which involves the adenosine triphosphate-fueled enzymatic oxidation of luciferins. Similarly, blue petaled Bark namely, Blue hydrangea, Cornflower, Morning glory, and Speedwell contains blue metal-anthocyanin supramolecular complexes and polyacylated anthocyanins are unstable pigment therefore difficult to synthesize and apply. Red beetroots comprise antioxidant betalain widely utilized as a food colorant [27]. Therefore, a quasibetalain, derived from betalamic acid with stretched $\pi$ - conjugation was obtained by Freitas, et al. The 1,11-diazaundecamethinium Beetblue chromophore is ethyl acetate acid-catalyzed by coupling of betalamic acid, extracted from beetroot juice and the carbon nucleophile 2,4-dimethylpyrrole, refer to Figure 88 [27].

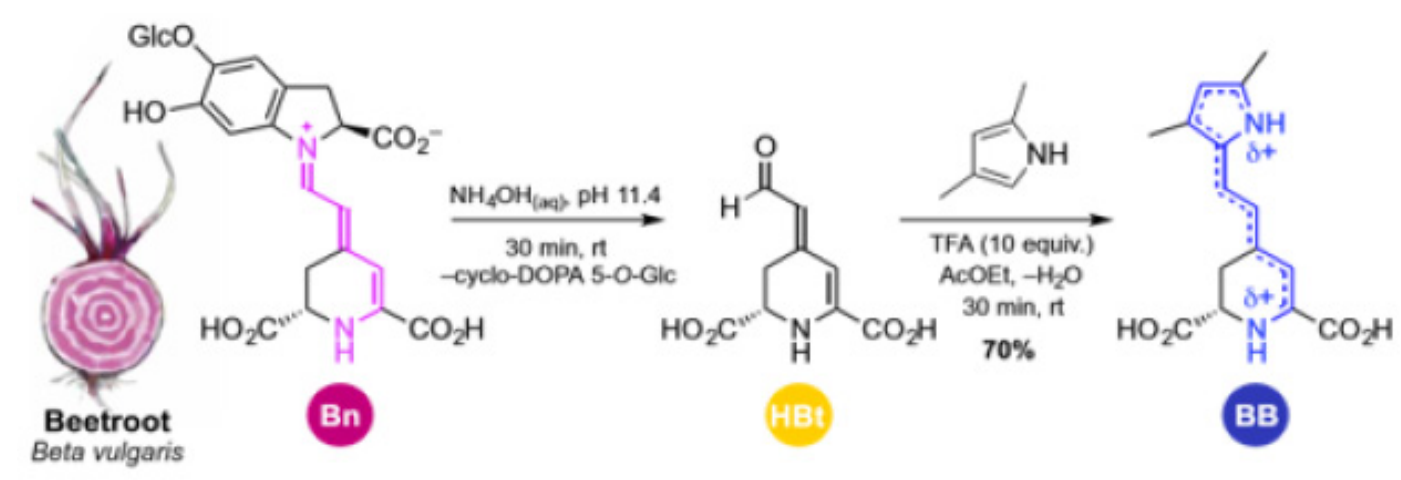

Figure 88: Synthesis of Beetblue chromophore [27].

The obtained Beetblue chromophore is highly water-soluble, stable, and could be applied on varied substrates as shown in Figure 89.

The toxic dyes produce singlet oxygen that damages cells. However, Beetblue was examined to be safe for human hepatic and retinal pigment epithelial cells. Beetblue does not affect zebrafish embryonal growth thus safe as concluded by Freitas et al., The study is remarkable and a prelude to enormous possibilities of research and development in green chemistry with plant-based renewable colors. 


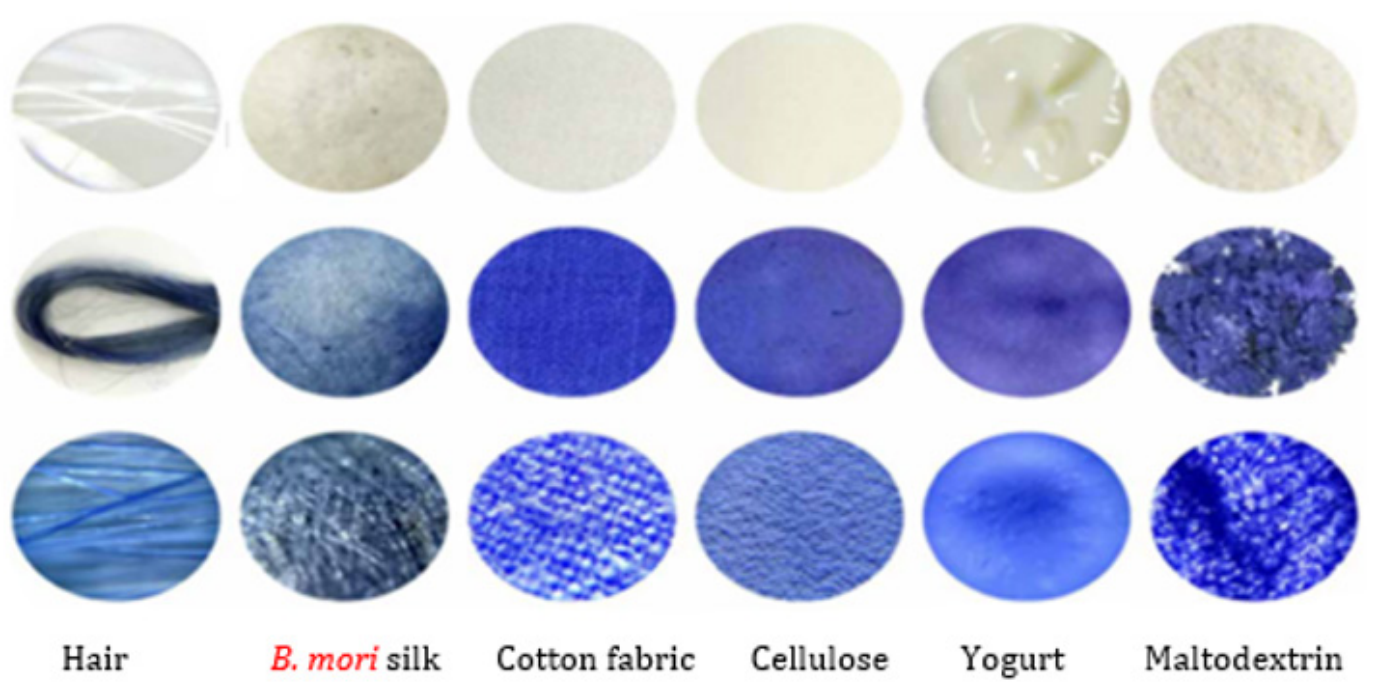

Figure 89: Beetblue on varied substrates [27].

The innovations with natural colors are beyond imaginations and the functionality of plant phytochemicals benefiting the wearers' health when in adherence to textiles is miraculous. A massively untapped resource ought to be cultivated for the sustainable future of the textile industry and would prodigiously benefit human and environmental health alike. Subsequently, to propel waterless technology herbal biomaterials are instigated for experiments with digital print technology. Implementation of herbal biomaterials for functional and sustainable textiles call in for enhanced responsibility towards protecting biodiversity and wise land planning if it to meet the growing demand [18]. Nevertheless, mass production, it could still massively and strongly contribute to sustainability in textiles. The plant phytochemicals play a subtle, strong, and vital role in the ecosystem.

\section{Conclusions}

The potential paper takes a holistic approach towards the subject wherein the idea is not to critic synthetic textile fibers and colors, on the other hand, to cohort the lens towards change in attitude at each step of textile production which is a chain reaction and circular in approach. The logic is to adapt to the principle of nature and back to nature which resonates with our creation. To accentuate our best compatibility with nature. In the words of Sir. A. Einstein 'Look deep into nature and you will understand everything better'. The biomimicry is what we learn from nature and acknowledge our deep connections to the biosphere [28]. The slow cyclic nature impels slow 'From nature, back to nature' principle for slow, steady, and winning economy, refer to Figure 90.

Hence, it is imperative to note that the natural plant-based biomaterials for textile applications are non-toxic and safe. They are predominantly synthesized from natural fruits, flowers, stems, leaves, barks grasses, seeds, roots, trees, and berries. The molecules of natural origin are biodegradable. Whereas the molecules of synthetic chemicals lack biodegradability and remain back in the environment releasing toxic fumes [29]. Moreover, the scientific research investigations accomplished on natural colors from plants indicate no possible health treat or toxicity to humans and animals alike [11].

Also, to further reinforce the sustainability agenda herbal materials for textile applications are often from surplus leaves, stems, twigs, flowers, fruits, fruit peels, and barks with no harm to the environment. Surplus water after fabric treatment is a zerocarbon footprint and could be recycled and reused. If discharged into the surrounding ground soil or water bodies, it causes no damage to aquatic life, environment, or human health therefore safe to be utilized for processing textile substrates. Still, to evident the theory, several types of research are being proposed examples of which are cited in the overview presented (Figure 90).

\section{Future Work}

Reflections from the overview recommend the most significant aspects that would propel sustainable textiles are as follows.

\section{Circular economy}

European commission in 2019 identified the textile clothing sector to be a priority in a sustainable product category as it massively contributes to the circular economy. European Environment Agency has set forth a strategic framework and corporate replicas to govern environmental and social burdens from textile consumption and production a short illustration of it is given in Figure 91. The policy's goals are to circumvent hazards from synthetic fossil-based textile production, greenhouse gas emissions, synthetic chemicals toxicity, and water effluents from textile industries. Sustainable plasma surface modification techniques and ecological digital print technology with herbal inks could occupy future research and developments for desirable sustainable outcomes. Eventually propelling the textiles oriented circular economy [18]. 


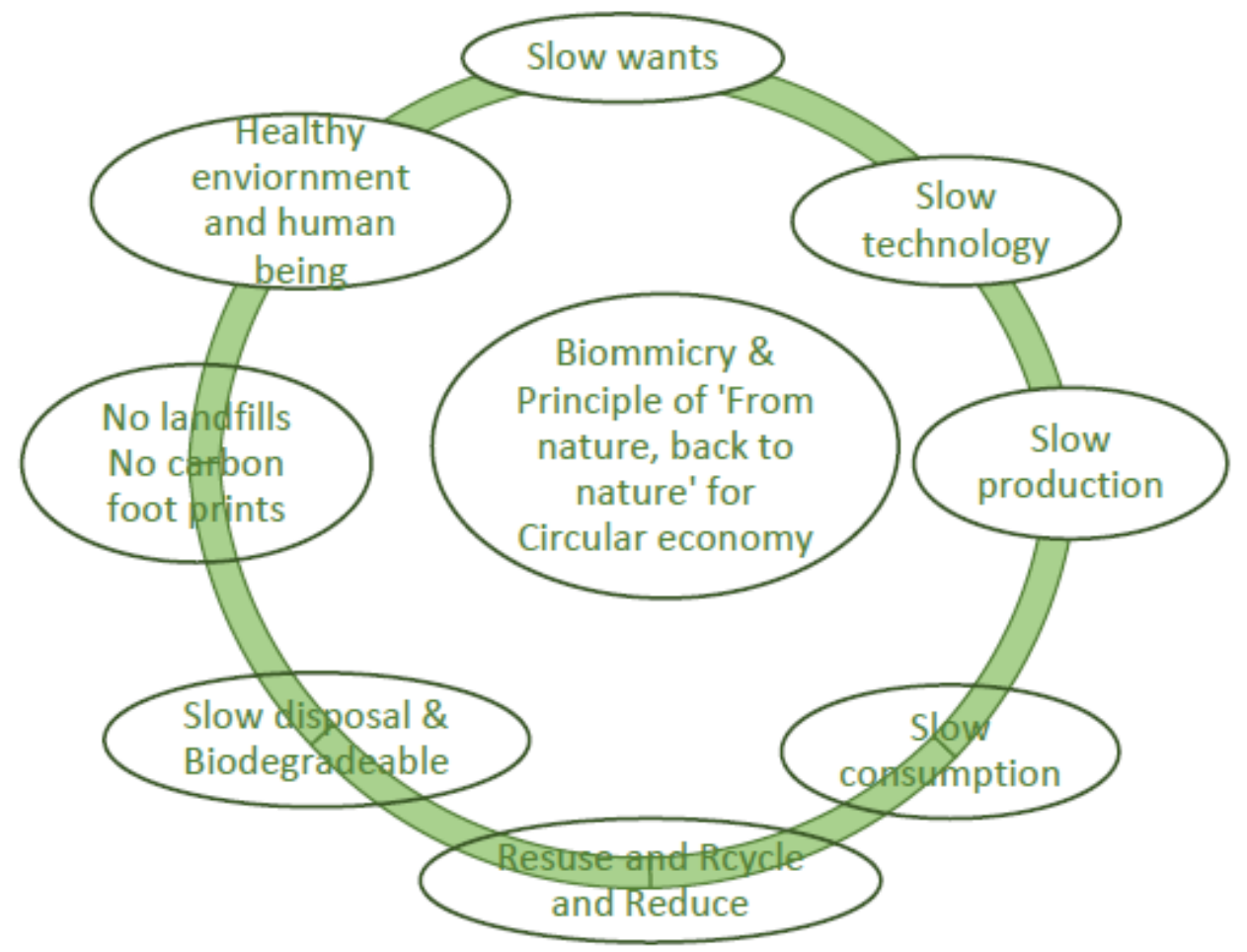

Figure 90: Biomimicry \& 'From Nature Back to Nature' Principle for Circular Economy.

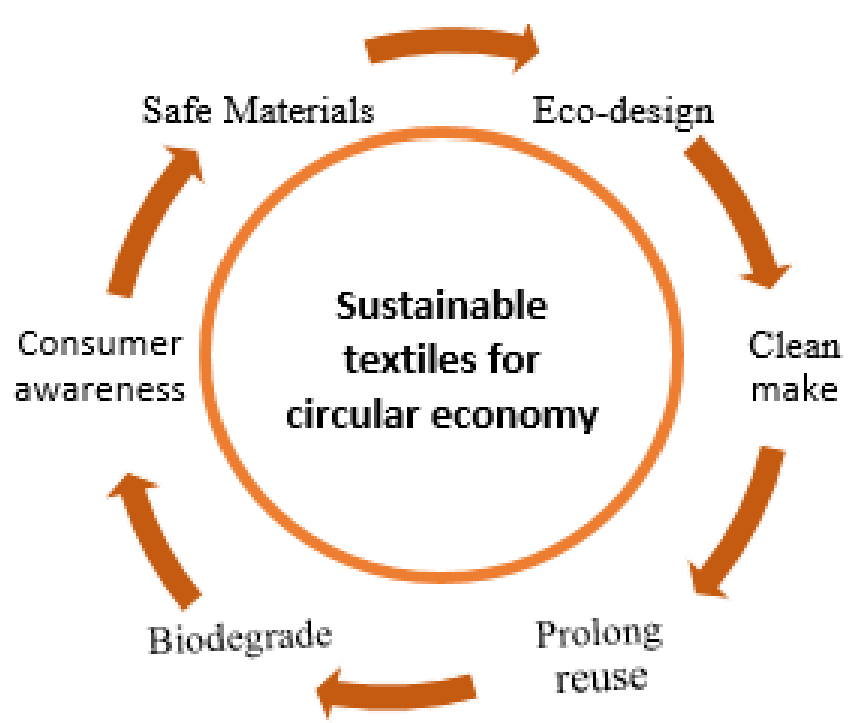

Figure 91: Sustainable textiles for the circular economy [18].

Consequently, $11 \%$ of market growth demand for natural dyes, pigments, and additives are predicted for 2025. To deliver the growing demand it would be essential to expand the color palette with herbal colors, adapt to ecological technology and software compatible with herbal colors, and rigorously affirm the functional properties of herbal materials by performing the required in-vitro and in-situ clinical studies. The ecological approach impels a circular economy (Figure 91).

\section{Multi-disciplinary research approach}

The review paper herein reinforces an interdisciplinary research approach for holistic outcomes. The multi-disciplinary research methodology cultivated and nurtured at the individual, national, and international levels would reap multiple gains for a higher good. An example of the same is illustrated in Figure 92 and elaborated as follows. 


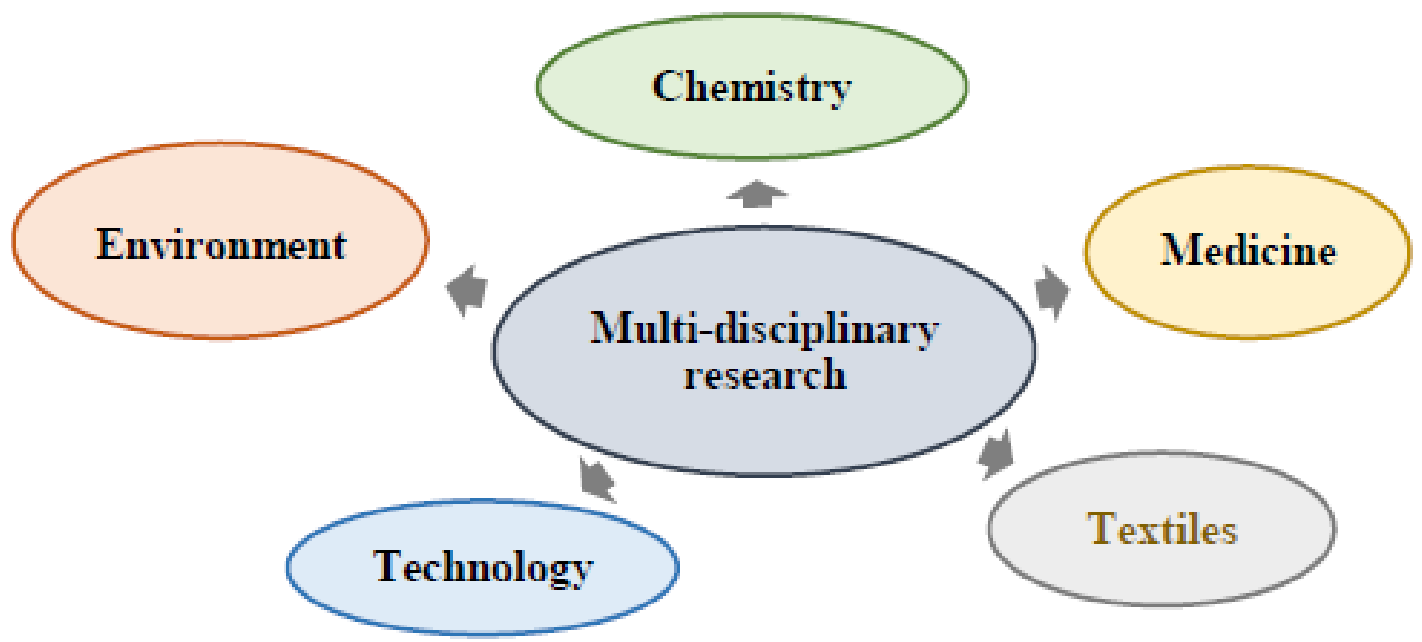

Figure 92: The multi-disciplinary research approach for global thriveability [18].

A multi-disciplinary research approach instigated herein investigates sustainable functional biomaterial for ecologically printed textiles with aesthetic merit. The envisaged engineering of fabrics essentially involves five fields in research as listed below wherein each field participates and profits propelling global thriveability.

Chemistry, the chemistry of plant phytochemicals of plantbased colors on the textile substrate play a subtle and vital role in research.

Medicine, UV protection, and the anti-microbial properties of the plant-based colors applied to the textile substrate are accentuated in research.

Textiles, the biomaterial engineering of plant-based colors on the textile substrate is primarily dealt with within the research.

Technology, ecological digital print technology, and plasma surface modification technology are adopted in research for sustainable outcomes.

Environment, the herbal formulations for textile application by sustainable technologies are assessed on life cycle assessment software befitting the environmental specifications.

It is imperative to note that the natural plant-based biomaterials for textile applications are non-toxic and safe. They are predominantly synthesized from natural fruits, flowers, stems, leaves, barks grasses, seeds, roots, trees, and berries. The molecules of natural origin are biodegradable. Whereas the molecules of synthetic chemicals lack biodegradability and remain back in the environment releasing toxic fumes.

To sum up the role and the potential of green chemistry for ecological and therapeutic textiles are enormously diverse and copiously existing in nature. Subsequently, with the commitment to protect biodiversity on earth and sustain our interdependent relationship with nature we ought to promulgate afforestation. Thriveability is justified scientifically for global enrichment.

\section{Biomimicry}

Biomimicry is an inspiration from nature as manifested herein. There are several species of wild herbs and shrubs, also weeds and grasses available in surplus, refer to Figure 93. These resources from the forest were recently experimented with by Griffiths, the author mordanted grey and white Romney wool skeins with traditional mordant alum and cream of tartar. Table 6 shows the wild species utilized for processing wool and the colors obtained. Natural coloration is an ancient art that could massively contribute towards sustainability irrespective of mass production. Also, the traditional art and craft natural dye artisans require to be supported by the legislative framework and academics [30]. Unlimited resources and scope of development opine the author (Figure 93).

Table 6: Colors were obtained on wool skeins with wild weeds, shrubs, and grasses [30].

\begin{tabular}{|c|c|c|}
\hline Wild Weeds, Shrubs \& Grasses & Grey Wool & White Wool \\
\hline Solidago (Goldenrod) & Olive Grey & Golden Yellow \\
\hline Andropogon gerardii (Big Bluestem Grass) & Yellow & Yellow \\
\hline Persicaria tinctoria (Indigo) & Citrine Green & Citrine Green \\
\hline Impatiens capensis (Orange Jewelweed) & Pink & Pink \\
\hline
\end{tabular}




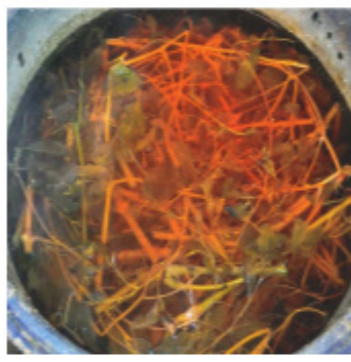

a) Jewelweed dyebath

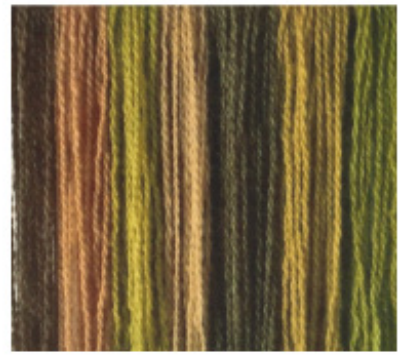

b) Wool skeins dyed with colors from weed, shrubs \& grasses

Figure 93: a) Jewelweed dyebath and b) wool skeins colored with natural dyes [30].

Similarly, Cia Wedin conjures up the fact that the Scandinavian style abides by certain values. One is environment-friendly production and craftsmanship which has a long tradition in the Nordic countries. The Scandinavian style predominantly works with natural materials. One of the traditional and common material in Nordic design is leather however it is not as good as it was once thought upon. Leather production has proved to be toxic, it involves abuse to animals, the environment, and humans. Therefore, the author opines that sustainability in itself is not a concept, it can rather be considered as an ever-changing process, where we experience, reflect, and learn how to produce and use design to reach the goals of agenda 2030 for sustainable development [31].

Also, Danish Design G-uld, envision that the colors of the museum are truly the slow pace sustainable contemporary colors and they discovered the mental and emotional layer that builds up with our deep connection with colors from nature onto our natural organic fiber fabrics [32]. Traditionally everything was organic. We polluted with over industrialization and overconsumption involving massive utilization of non-sustainable fossil-based resources. Contemplatively we ought to reflect and adapt to alternative materials for global thriveability. However, not all the synthetic is appalling or could be replaced, those from which, as listed in the M-RSL (Given in Part-1 article), resulting in enormous pollution and health problems require to be substituted. The negative impacts of overconsumption, over industrialization, and non-biodegradability due to fossil-based materials in the textile chain is to be addressed. The biomimicry - and inspirations from nature, could greatly help us in the same.

To sum up, realistically we do not require the clothes, colors, materials like plastic and accessories having a lifespan much longer than the humans themselves live particularly those generating emissions and effluents and easily harming the environment and human health alike. The logic is not to simply criticize or aim for replacing fossil-based derivatives. The requirement is to regain the lost balance. To reflect on laws of nature, laws of physics, and laws of biology to sustain the planet and the life on it. And hope lies in harmony with nature. Circular materials for the circular economy look as if inherently compatible to conserve our interdependent relationship with nature for global sustainability.

\section{Acknowledgment}

The author is grateful to the anonymous funder for sponsoring sustainability-based research for global cause and benefits.

\section{Conflict of Interest}

Authors declare no conflict of interest.

\section{References}

1. Greenpeace International (2018) Destination Zero: seven years of Detoxing the clothing industry. Greenpeace.e.V, Hamburg.

2. Martinez S (2017) Textile factories in Indonesia pollute water in Cikijing River, a tributary of Citarum River, Indonesia. Environmental Justice Atlas.

3. (2020) Stichting ZDHC Foundation, "Roadmap to change," Roadmap to zero, 2020.

4. Greenpeace International (2013) Toxic Threads: Polluting Paradise, A story of big brands and water pollution in Indonesia. Greenpeace International, UK.

5. Kate F (2008) Sustainable Fashion and Textiles Design Journeys, UK: Earthscan.

6. Prasanna P Gunathilake KD, Somathilaka Ranaweera KD, Rupasinghe Vasantha HP (2018) Effect of Different Cooking Methods on Polyphenols, Carotenoids and Antioxidant Activities of Selected Edible Leaves. Antioxidants 7(9): 117.

7. Manimozhi R, Kanakarajan S (2017) Natural Dye from Torenia SP Flower for Colouring Silk Yarn using Biomordants-An Ecofriendly Approach. Journal of Chemical and Pharmaceutical Research 9(11): 17-26.

8. Thakker AM, Sun D (2020) Sustainable processing of plant-based colors on bamboo, banana and merino wool fibers.

9. Buckley B (2016) Does Cooking Destroy Nutrients and Phytochemicals in Cauliflower and Broccoli? Nutrition Carnival.

10. (2020) United Nations, "United Nations Sustainable Developments," United Nations.

11. Shukla D, Vankar P (2019) New trends in natural dyes for textiles. ( $1^{\text {st }}$ edn), Woodhead Publishing, UK, p.368.

12. Cardon D (2007) Natural Dyes - Sources, Tradition, Technology and Science. Archetype Publications Ltd, London, UK.

13. (2020) Merriam-Webster, Incorporated, “Merriam Webster Since 1828," 2020 Merriam-Webster, Incorporated. 
14. (2020) Oxford University Press, "Lexico," Dictionary.com and Oxford University Press.

15. PerkinElmer Informatics, “ChemDraw," PerkinElmer Inc, USA, 1998 2020 .

16. (2020) National Center for Biotechnology Information. Pubchem Explore Chemistry, National Library of Medicine.

17.(2020) naturalmedicinefacts.info, "Natural medicine facts," naturalmedicinefacts.info 2015.

18. Thakker AM, Sun D (2020) Sustainable plant-based bioactive materials for functional printed textiles. The Journal of The Textile Institute.

19. Vaideki K, Jayakumar S, Rajendran R, Thilagavathi G (2008) Investigation on the effect of RF air plasma and neem leaf extract treatment on the surface modification and antimicrobial activity of cotton fabric. Applied Surface Science 254(8): 2472-2478.

20. Chandrasekaran K, Senthilkumar M (2019) Synergic anti-bacterial effect of Curcuma aromatica Salisb and Ocimum tenuiflorum Linn herbal extract combinations on treated cotton knitted fabrics against selective bacterial strains. Indian Journal of Fiber \& Textile Research 44(3): 344 351.

21. Bonet-Aracil MA, Pablo DG, Eva BB, Natividad S, Montoro A, et al. (2016) UV protection from cotton fabrics dyed with different tea extracts. Dyes and Pigments 134: 448-452.

22. Chengaiah B, Rao K, Kumar K, Alagusundaram M, Madhusudhana C (2010) Medicinal importance of natural dyes. International Journal of Pharm Tech Research 2(1): 144-154.

23. Kole SS, Gotmare VD, Athawale RB (2019) Novel approach for the development of eco-friendly antimicrobial textile materials for health care application. The Journal of the Textile Institute 110(2): 254-266.
24. Janarthanan M, Senthilkumar M (2019) Ontogenesis of textile face mask using cotton fabric by treating with red seaweeds extract for cosmetotextiles applications. The Journal of the Textile Institute 110(7): 959-971.

25. Ramanarayanan R, Nijisha P, Niveditha CV, Sindhu S (2017) Natural dyes from red amaranth leaves as light-harvesting pigments for dyesensitized solar cells. Materials research bulletin 90: 156-161.

26. Devarayan K, Suhk Kim B (2015) Reversible and universal pH sensing cellulose nanofibers for health monitor. Sensors and Actuators B: Chemical 209(31): 281-286.

27. Freitas Dörr BC, Machado CO, Pinheiro AC, Fernandes AB, Dörr FAet al. (2020) A metal-free blue chromophore derived from plant pigments. Sci Adv 6(14): eaaz0421.

28. Glover S (2020) Biomimicry - Why fashion needs to learn from nature? Ecotextile News: 40 .

29. Sharma D, Singh R, Dighe B (2018) Chromatographic study on traditional natural preservatives used for palm leaf manuscripts in India. Restaurator International Journal for the Preservation of Library and Archival Material 39(4): 249-264.

30. Griffiths C (2020) An Afternoon of Dyeing. The Journal of Weavers, Spinners and Dyers 275: 12-14.

31. Wedin C (2020) What goes around comes around - Scandinavian style and sustainability. Selvedge - The fabric of your life: Nordic: 22-24.

32. Danish Design Guld (2020) Complementary colors. Selvedge - The fabric of your life: Nordic: 82-85. 